\title{
Comparative fiber property and transcriptome analyses reveal key genes potentially related to high fiber strength in cotton (Gossypium hirsutum L.) line MD52ne
}

Md S. Islam', David D. Fang ${ }^{1}$, Gregory N. Thyssen², Chris D. Delhom ${ }^{3}$ Yongliang Liư ${ }^{3}$ and Hee Jin Kim*

\begin{abstract}
Background: Individual fiber strength is an important quality attribute that greatly influences the strength of the yarn spun from cotton fibers. Fiber strength is usually measured from bundles of fibers due to the difficulty of reliably measuring strength from individual cotton fibers. However, bundle fiber strength (BFS) is not always correlated with yarn strength since it is affected by multiple fiber properties involved in fiber-to-fiber interactions within a bundle in addition to the individual fiber strength. Molecular mechanisms responsible for regulating individual fiber strength remain unknown. Gossypium hirsutum near isogenic lines (NILS), MD52ne and MD90ne showing variations in BFS provide an opportunity for dissecting the regulatory mechanisms involved in individual fiber strength.
\end{abstract}

Results: Comprehensive fiber property analyses of the NILs revealed that the superior bundle strength of MD52ne fibers resulted from high individual fiber strength with minor contributions from greater fiber length. Comparative transcriptome analyses of the NILs showed that the superior bundle strength of MD52ne fibers was potentially related to two signaling pathways: one is ethylene and the interconnected phytohormonal pathways that are involved in cotton fiber elongation, and the other is receptor-like kinases (RLKs) signaling pathways that are involved in maintaining cell wall integrity. Multiple RLKs were differentially expressed in MD52ne fibers and localized in genomic regions encompassing the strength quantitative trait loci (QTLS). Several candidate genes involved in crystalline cellulose assembly were also up-regulated in MD52ne fibers while the secondary cell wall was produced.

Conclusion: Comparative phenotypic and transcriptomic analyses revealed differential expressions of the genes involved in crystalline cellulose assembly, ethylene and RLK signaling pathways between the MD52ne and MD90ne developing fibers. Ethylene and its phytohormonal network might promote the elongation of MD52ne fibers and indirectly contribute to the bundle strength by potentially improving fiber-to-fiber interactions. RLKs that were suggested to mediate a coordination of cell elongation and SCW biosynthesis in other plants might be candidate genes for regulating cotton fiber cell wall assembly and strength.

Keywords: Bundle fiber strength, Cellulose assembly, Ethylene, Gossypium hirsutum, Individual fiber strength, Near-isogenic line, Receptor-like kinase

\footnotetext{
* Correspondence: heejin.kim@ars.usda.gov

${ }^{1}$ USDA-ARS, Southern Regional Research Center, Cotton Fiber Bioscience

Research Unit, New Orleans, LA 70124, USA

Full list of author information is available at the end of the article
} 


\section{Background}

Cotton (Gossypium spp.) fiber is the most important natural fiber in the textile industry [1]. Physical properties such as strength, length, maturity (degree of thickness), and fineness determine the value and quality of cotton fibers and the yarn spun from them. With the advent of modern high speed spinning machinery, which produces a quality yarn in a cost effective way, the demand for stronger fiber has increased in the highly competitive global textile market. During the past 2 decades, the fiber strength of US cotton has gradually improved through breeding [2], however, the pace of improvement is restricted by our limited knowledge of fiber strength development.

Both cotton producers and processers usually measure fiber strength from a bundle composed of thousands of individual fibers. The strength simply refers to the force required to break fibers [1,3]. The bundle fiber strength (BFS), which is also called fiber tenacity, is measured by an automated high-volume instrument (HVI) designed to measure the market value of cotton fibers in efficient and rapid ways. Currently, the Agricultural Marketing Service (AMS) of the United States Department of Agriculture (USDA) separates cotton fibers into different classes ranging from the lowest $(23.0 \mathrm{~g} /$ tex or below) to the highest class ( $31.0 \mathrm{~g} /$ tex and above) based on the BFS values measured by the HVI. A stelometer is used to measure the BFS value for a relatively small amount of fibers [1, 3-5]. Due to the inherent variability among fibers within a bundle, two fiber bundles of the same weight do not have the same number of fibers. Thus, the actual BFS is determined in grams per tex (g/tex) in which the breaking force $(\mathrm{g})$ is standardized by the linear density or fineness $($ tex $=\mathrm{g} / \mathrm{km})$. Hence, high BFS $(\mathrm{g} / \mathrm{tex})$ is obtained by either increasing the breaking force (g) or deceasing the fineness value (tex).

The cotton industry has been using fiber properties determined by the HVI and Advanced Fiber Information System (AFIS) as parameters for predicting yarn quality and selecting the right raw cotton materials to produce different qualities of yarns. However, several textile papers have reported that the BFS data of cotton fibers were inadequate to predict yarn strength due to insignificant correlation between the cotton BFS and the yarn strength $[4,6,7]$. The BFS values are regulated by intrinsic fiber strength and other fiber properties which affect fiber-to-fiber interactions within a bundle [1, 3, 4, 8-10]. Fiber length values that promote fiber interactions within a bundle impact the BFS values [11]. Fiber thicknessrelated properties including micronaire (MIC), maturity ratio (MR), and fineness values (tex) also affect bundle strength of cotton fibers since they determine the number of individual fibers in a fiber bundle [9].

The intrinsic fiber breaking force value is correlated with yarn strength and can be measured from individual fibers by Mantis or Favimat instruments [1, 3, 8, 9, 11]. Average breaking force $(\mathrm{cN})$ obtained from several hundred individual fibers was not affected by other bundle fiber properties and was reported as one of the most important factors in determining the strength of the yarn spun from those fibers $[4,12,13]$. Despite the usefulness of the individual fiber properties, the Mantis and Favimat instruments, which require laborious processes, have not been previously utilized in cotton genetics and genomics research.

Gossypium hirsutum germplasm NILs, MD52ne and MD90ne were developed through backcross breeding [14]. MD90ne is the recurrent parent and MD52ne is a $\mathrm{BC}_{6}$ high-BFS selection. MD52ne contains $10 \%$ higher BFS values, $22 \%$ less short fibers, and $7 \%$ greater fiber length than its NIL, MD90ne [15]. The stronger BFS trait of MD52ne was suggested to be controlled by a small number $(\leq 2)$ of genes [15]. By using the prototype of cotton oligonucleotide microarray chips that was developed from cotton expressed sequence tags (ESTs) [16], we previously observed a temporal up-regulation of secondary cell wall (SCW) biogenesis genes in MD52ne fiber at the transition from fiber elongation to secondary cell wall (SCW) biosynthesis as compared with MD90ne [17]. Another group also suggested that SCW biogenesis-related genes might contribute to the fiber strength of G. hirsutum chromosome introgression lines (CSILs) carrying chromosomal segments from G. barbadense whose fibers are longer, stronger, and finer than their recurrent parent G. hirsutum TM-1 [18]. The G. hirsutum CSILs were used for identifying potential genes responsible for fiber length [19] in addition to fiber strength [18]. Since the fiber length and fineness affecting fiber-to-fiber interactions within a bundle can modulate the BFS values of the cotton NILs [17] and CSILs [18], it is not clear if the SCW biogenesis-related genes are involved in regulating individual fiber breaking force and/or other bundle fiber properties. Indeed, the same SCW biogenesis-related genes were identified as differentially expressed genes (DEGs) that may be involved in molecular mechanisms of controlling fiber length [19] and fiber thickness-related properties $[20,21]$ in addition to the BFS $[17,18]$. Therefore, it remains to be answered which candidate genes are really involved in individual fiber breaking force and therefore yarn strength.

In this research, we measured fiber properties of mature and developing fibers of MD52ne, MD90ne, and their $F_{2}$ progenies using both novel and conventional methods including Favimat, cross-section image analysis microscopy, attenuated total reflectance Fourier transform infrared spectroscopy (ATR-FTIR), HVI, Stelometer, AFIS, and gravimetric fineness methods. The extended fiber property analyses showed that the superior BFS of MD52ne fibers resulted primarily from a higher individual fiber breaking 
force with a minor contribution from increased fiber length. Comparative transcriptome analyses of the NILs suggested that the superior BFS of MD52ne fibers was potentially related to ethylene pathway-directed fiber elongation and enhanced cell wall integrity due to the activity of receptor-like kinase (RLK) signaling pathways.

\section{Results}

Comparisons of bundle fiber properties between mature MD52ne and MD90ne fibers

Fiber property analysis by HVI showed that the BFS of mature MD52ne fibers was significantly greater (19 $25 \%)$ than that of MD90ne fibers grown in two separate fields that substantially differ in geographic location and environmental conditions (Table 1). In addition, upper half mean length (UHML) of the MD52ne fiber was longer (5 6\%). Average fiber elongation values of MD52ne were lower than those of MD90ne but was only statistical significant at one location. No significant differences in the MIC values were observed between the MD52ne and MD90ne grown in both locations.

\section{Correlation of bundle fiber strength with other fiber properties}

To determine how the BFS values of the NILs were affected by other physical properties involved in fiber-tofiber interactions, we developed an $\mathrm{F}_{2}$ population of 384 progeny plants derived from a cross between MD52ne and MD90ne. Among the $F_{2}$ progenies, the BFS values showed a Gaussian distribution with a broad range (31.99 and $42.66 \mathrm{~g} /$ tex) (Fig. 1a). Other fiber properties including UHML length $(27.82 \sim 32.48 \mathrm{~mm})$, elongation (4.78 6.45\%), MIC (4.39 5.78), maturity ratio $(0.979 \sim 1.078)$, and fineness $(172.0 \sim 215.0 \mathrm{mtex})$ measured by HVI and AFIS showed Gaussian distributions among the $F_{2}$ progenies so we were able to determine correlations of the BFS with other fiber physical properties.
Table 2 showed that the BFS values of the NILs were affected by fiber length (UHML, mm) and micronaire (MIC) measured by HVI, and the maturity ratio (MR) and fineness (mtex) measured by AFIS. The correlation coefficient values $(r)$ by Pearson's method [22] showed that the BFS value was positively correlated with UHML, MIC, MR, or fineness, whereas the BFS showed no significant correlation with $\mathrm{FE}$. The $R^{2}$ values suggested that BFS variances were slightly affected by UHML (12.9\%), whereas they had little effect from fiber thickness-related properties such as MIC (1.2\%), MR (2.9\%), and fineness (1.2\%).

\section{Comparisons of individual fiber properties between mature MD52n and MD90ne fibers}

To minimize the influences of other physical fiber properties on fiber strength, we first screened for the NIL plants that had similar fiber properties except the BFS values. Among the selected NIL plants having a significant variation (24\%) of BFS values, we identified each cotton MD52ne and MD90ne line having an almost identical MIC value (4.931) representing a combination of fiber maturity ratio and fineness. The fiber properties measured by AFIS in addition to the HVI confirmed that there was no significant variation in the fiber thickness-related properties (MIC, MR, and fineness) but a significant difference (5\%, UHML) in fiber length between the selected NILs (Table 3). The average breaking force of individual MD52ne fibers was significantly greater $(22.4 \%)$ than that of individual MD90ne fibers (Table 3). The distribution curves showed great variation of breaking forces among individual fibers of each NIL and a similar distribution range of breaking force between the individual MD52ne fibers $(0.93 \sim 11.25 \mathrm{cN})$ and MD90ne fibers $(0.62 \sim 11.27 \mathrm{cN})$ (Fig. 1b). Stronger fibers with higher $\mathrm{cN}$ values were more prevalent in MD52ne than MD90ne.

Table 1 Comparisons of HVI bundle fiber properties between MD52ne and MD90ne

\begin{tabular}{|c|c|c|c|c|c|}
\hline \multirow[t]{2}{*}{ Fiber properties } & \multirow[t]{2}{*}{ Cotton Fields } & \multirow[t]{2}{*}{ MD52ne } & \multirow[t]{2}{*}{ MD90ne } & \multirow[t]{2}{*}{ MD52ne/MD90ne } & \multirow{2}{*}{$\begin{array}{l}t \text { test } \\
p \text { value }\end{array}$} \\
\hline & & & & & \\
\hline \multirow[t]{2}{*}{ BFS (g/tex) } & Stoneville, MS & $39.95 \pm 1.84$ & $33.61 \pm 1.61$ & 1.19 & $<0.0001^{* * *}$ \\
\hline & New Orleans, LA & $39.69 \pm 2.69$ & $31.79 \pm 2.87$ & 1.25 & $0.002^{* *}$ \\
\hline \multirow[t]{2}{*}{ UHML (mm) } & Stoneville, MS & $31.24 \pm 0.51$ & $29.72 \pm 1.02$ & 1.05 & $<0.0005^{* * *}$ \\
\hline & New Orleans, LA & $31.50 \pm 0.76$ & $29.85 \pm 0.51$ & 1.06 & $0.0059^{* *}$ \\
\hline \multirow[t]{2}{*}{ FE (\%) } & Stoneville, MS & $5.45 \pm 0.18$ & $5.96 \pm 0.26$ & 0.91 & $<0.0001^{* * *}$ \\
\hline & New Orleans, LA & $4.74 \pm 0.13$ & $4.94 \pm 0.60$ & 0.96 & 0.487 \\
\hline \multirow[t]{2}{*}{ MIC } & Stoneville, MS & $4.94 \pm 0.29$ & $5.01 \pm 0.07$ & 0.99 & 0.468 \\
\hline & New Orleans, LA & $5.86 \pm 0.79$ & $5.25 \pm 0.23$ & 1.11 & 0.136 \\
\hline
\end{tabular}

Each value is the mean \pm SD. Statistical significance was shown at the probability levels under $0.01^{* *}$, and $0.001^{* * *}$. HVI, High Volume Instrument; BFS, bundle fiber strength; UHML, upper half mean length; UI, uniformity index; FE, fiber elongation; MIC, micronaire 
A

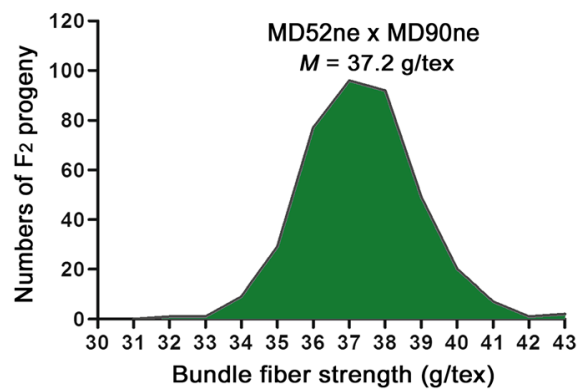

B

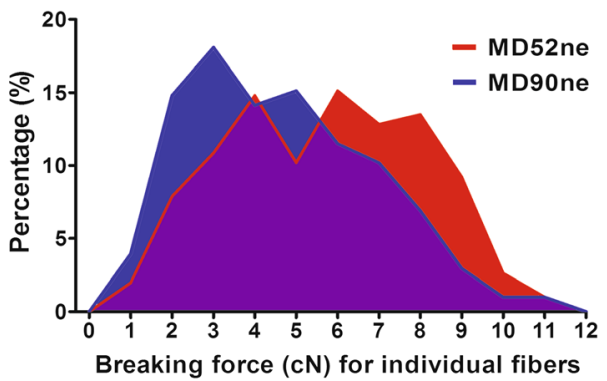

Fig. 1 Fiber property analyses of NILs and their $F_{2}$ progeny. a Frequency distribution of bundle fiber strength in an $F_{2}$ population from a cross between MD90ne and MD52ne. Average bundle strength value of each $\mathrm{F}_{2}$ progeny was obtained from five replicates by $\mathrm{HVI}$. Minimum, median $(M)$, and maximum bundle fiber strengths were 31.99, 37.22, and $42.66 \mathrm{~g} /$ tex among $384 \mathrm{~F}_{2}$ progenies. b Comparisons of breaking force distributions of individual fibers from MD52ne and MD90ne fibers. Breaking forces (cN) of 303 individual fibers were determined from mature MD52ne and MD90ne fibers having similar fiber maturity with an identical MIC value (4.931). A 13 mm length gauge was used for breaking fiber with Favimat instrument

\section{Comparisons of fiber properties between developing MD52ne and MD90ne fibers}

To determine when variations of fiber properties occurred during cotton fiber development between the two NILs, we measured fiber physical properties from developing fibers at ten different developmental time points $(10,13,15,17,20,24,28,33,37$, and 44 DPA) covering entire developmental stages (Fig. 2). The growth rates of actively elongating MD52ne and MD90ne fibers declined at 20 DPA (Fig. 2a). Statistical analyses using $t$ test showed no significant variances on the average lengths of the developing fibers younger than 24 DPA between the NILs. Average fiber lengths of developing MD52ne fibers at $28 \mathrm{DPA}$ and older were significantly longer ( $p$ value, 0.0012$)$ than those of developing MD90ne fibers at the corresponding time points. At around $15 \mathrm{DPA}$, fibers entered a transition phase with both SCW cellulose biosynthesis and fiber elongation (Fig. 2b). Crystallinities of developing MD52ne and MD90ne fibers were measured by an ATR-FTIR spectroscopy and its corresponding algorithm [23, 24]. The comparative plot showed low crystallinity $\left(C I_{\mathrm{IR}}\right)$ of the developing fibers between 10 and 15 DPA. The $C I_{I R}$ values increased rapidly to $17 \mathrm{DPA}$, and reached its peak at 37 DPA (Fig. 2b). The observation implied that the developing fibers at 10,13, and 15 DPA were composed of mainly primary cell wall $(\mathrm{PCW})$ containing a low crystallinity and the transition from elongation to SCW biosynthesis occurred from 15 to 17 DPA. SCW containing a high crystallinity was deposited on the developing fibers until 37 DPA of both NILs. There was no statistically significant difference in the $C I_{\mathrm{IR}}$ values of developing fibers between the two NILs during the entirety of the developmental stages (Fig. 2b). The bolls became fully developed and began to open at approximately 42 DPA. The mature fibers dehisced at 44 DPA. Fiber maturity ratio or degree of wall thickness $\left(M_{\mathrm{IR}}\right)$ was also assessed from developing MD52ne and MD90ne fibers by ATR-FTIR spectra (Fig. 2c) [23, 24]. The developing fibers at 17 DPA and younger showed little of the SCW cellulose that is mainly responsible for fiber maturity [25]. The $M_{\mathrm{IR}}$ values revealed that the developing fibers at 20 DPA and older consisted of SCW cellulose. During the SCW biosynthesis stage (20-33 DPA) of both NIL fibers, the $M_{\mathrm{IR}}$ values increased likewise (Fig. 2c). Cross-sectioned images of fully mature MD 52ne and 90ne fibers and their calculated MR showed similarities between the NILs (Fig. 3). The increasing pattern of fineness values (40.0 to 205.6 mtex) of developing MD52ne fibers from 20 to 44 DPA was almost identical to that (43.3 to 205.6 mtex)

Table 2 Correlations between bundle fiber strength and other fiber properties from the $F_{2}$ plant derived from a cross between MD52ne and MD90ne

\begin{tabular}{lllclll}
\hline Fiber properties & Measuring method & Sample numbers & Correlation coefficient $(r)$ & $R^{2}$ & $p$-value & Correlation with BFS \\
\hline UHML & HVI & 384 & 0.360 & 0.129 & $<0.0001^{* * *}$ & Yes \\
MIC & HVI & 384 & 0.108 & 0.012 & $0.0343^{*}$ & Yes \\
MR & AFIS & 380 & 0.169 & 0.029 & $0.001^{* * *}$ & Yes \\
Fineness & AFIS & 380 & 0.110 & 0.012 & $0.0325^{*}$ & Yes \\
FE & HVI & 384 & -0.049 & 0.002 & 0.340 & No \\
\hline
\end{tabular}

Average bundle fiber strength (BFS), fiber length (UHML), micronaire (MIC), maturity ratio (MR), fineness, and fiber elongation (FE) were calculated from five replicates of each $\mathrm{F}_{2}$ progeny plant. Linear regression and correlation data between bundle fiber strength and other fiber properties were determined by Graphad Prism 5.0. Statistical significance was shown at the probability levels under $0.05^{*}$, and $0.001^{* * *}$ 
Table 3 Comparisons of bundle and individual fiber properties between mature MD52ne and MD90ne fibers having identical MIC value

\begin{tabular}{|c|c|c|c|c|c|}
\hline \multirow[t]{2}{*}{ Measuring methods } & \multirow[t]{2}{*}{ Fiber Properties } & \multirow[t]{2}{*}{ MD52ne } & \multirow[t]{2}{*}{ MD90ne } & \multirow[t]{2}{*}{ MD52ne/MD90ne } & \multirow{2}{*}{$\begin{array}{l}t \text { test } \\
p \text { value }\end{array}$} \\
\hline & & & & & \\
\hline \multirow[t]{3}{*}{$\mathrm{HVl}$} & MIC & $4.931 \pm 0.02$ & $4.931 \pm 0.06$ & 1.00 & 1.0000 \\
\hline & BFS (g/tex) & $39.27 \pm 1.41$ & $31.66 \pm 1.19$ & 1.24 & $<0.0001^{* * *}$ \\
\hline & UHML (mm) & $31.01 \pm 0.79$ & $29.59 \pm 0.48$ & 1.05 & $0.0089^{* *}$ \\
\hline \multirow[t]{2}{*}{ AFIS } & Maturity ratio (MR) & $0.99 \pm 0.02$ & $0.97 \pm 0.01$ & 1.02 & 0.1963 \\
\hline & Fineness (mtex) & $171.67 \pm 6.11$ & $173.67 \pm 3.06$ & 0.99 & 0.6389 \\
\hline Favimat $^{a}$ & Breaking force (cN) & $5.67 \pm 2.34$ & $4.63 \pm 2.18$ & 1.22 & $<0.0001^{* * *}$ \\
\hline
\end{tabular}

${ }^{a}$ Average breaking force $(\mathrm{cN})$ was calculated from 303 individual fibers by Favimat. Each value is the mean \pm SD. Statistical significance was shown at the probability levels under $0.05^{*}, 0.01^{* *}$, and $0.001^{* * *}$. HVI, High Volume Instrument; AFIS, Advanced Fiber Information System; MIC, micronaire; BFS, bundle fiber strength

A

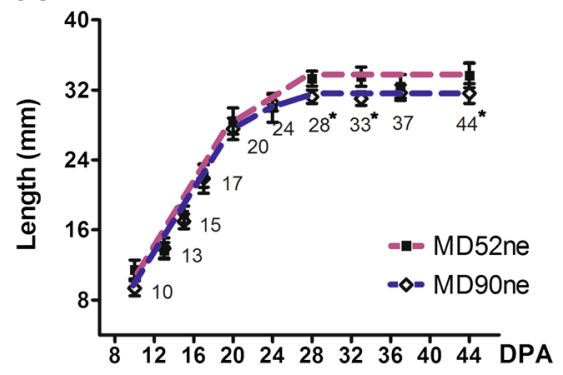

C

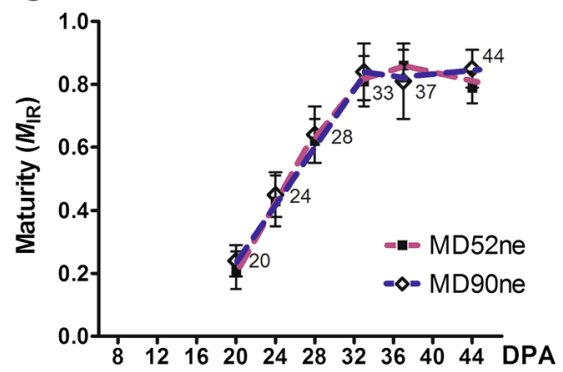

B

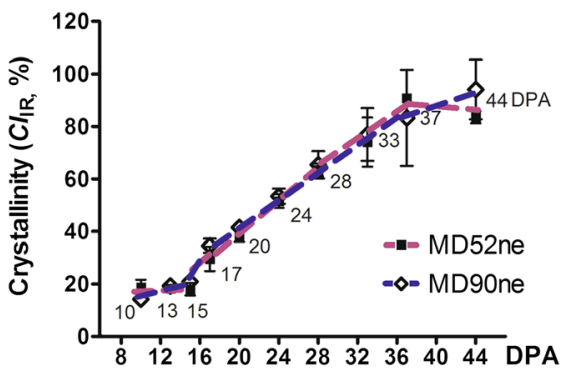

$\mathrm{D}$

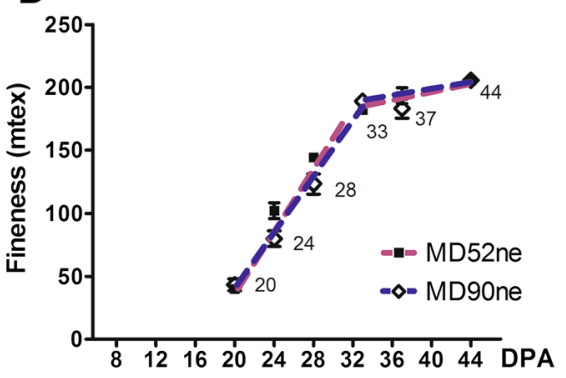

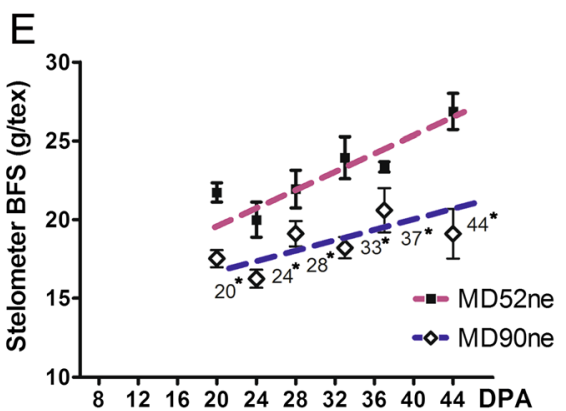

Fig. 2 Comparisons of fiber properties from developing MD52ne and MD90ne fibers. Developing fibers at nine different developmental time points $(10,13,15,17,20,24,28,33$, and 37 DPA) and full developed fibers at 44 DPA were collected from NILs grown side by side in replicated fields in New Orleans, LA in 2013. Asterisks next to the time points denote statistical significance. a Fiber length. Average lengths of developing fibers at different DPAs were calculated from 30 replicates. $\mathbf{b}$ Crystallinity. Average crystallinity $\left(C_{\mathbb{R}}\right)$ was determined from six replicates of ATR-FTIR spectra. c Maturity. Average fiber maturity $\left(M_{\mathbb{R}}\right)$ was determined from six replicates using ATR-FTIR. $\mathbf{d}$ Fineness. Three hundred fibers of $15 \mathrm{~mm}$ length were used for each replicate of gravimetric fineness analyses. Average fineness values were calculated from three replicates. e Bundle fiber strength. Average bundle fiber strength of developing fibers was measured from three replicates by Stelometer 


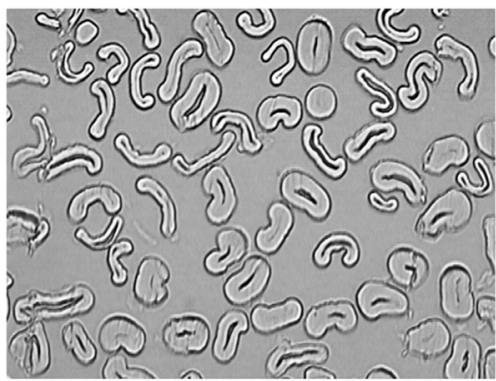

MD52ne

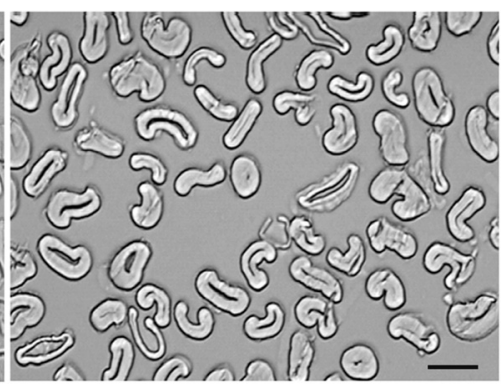

MD90ne

Fig. 3 Comparisons of fiber maturity ratios of mature fibers between MD52ne and MD90ne measured by image analysis microscopy. Circularity $\left(\theta=4 \pi A / P^{2}\right)$ representing the degree of fiber cell wall thickness was calculated from average wall area $(A)$ excluding lumen and perimeter ( $P$ ) of cross-sections of 300 fibers. The maturity ratios between MD52ne $(0.948 \pm 0.010)$ and MD90ne $(0.971 \pm 0.033)$ calculated from the circularities showed no significant variation ( $p$-value, 0.519). A scale bar represents $10 \mu \mathrm{m}$. MD52ne, MD90ne

of developing MD90ne fibers at the corresponding time points (Fig. 2d). Developing MD52ne fibers at 20 DPA and older were significantly ( $p$-value $<0.0008)$ stronger than developing MD90ne fibers at the corresponding DPAs (Fig. 2e). When the developing fibers reached 20 DPA, the BFS differences were clearly detected between MD52ne (21.7 g/tex) and MD90ne (17.5 g/tex). A stelometer, which requires relatively less fiber than the HVI, was used for measuring bundle strength of developing fibers. Neither the stelometer nor the HVI could measure BFS values from developing fibers that were younger than 20 DPA, because the sugary and sticky developing fibers cannot be individualized.

\section{Transcriptome analysis of developing fibers between MD52ne and MD90ne by RNA-seq}

To investigate the molecular basis for the superior fiber strength of MD52ne to MD90ne, whole genome comparative transcriptome analyses were performed with total
RNAs extracted from developing fibers between MD52ne and MD90ne. Based on the fiber property data obtained from developing fibers (Fig. 2), two different developmental time points, 15 DPA containing mainly PCW and 20 DPA containing both PCW and SCW, were compared between the NILs with two biological replicates.

The average number of raw reads per library obtained by paired-end Illumina sequencing ranged from 32.80 to $33.49,000,000$ reads (Table 4). The numbers of average raw reads were slightly higher in MD52ne in both time points than MD90ne. Of the raw reads, 80.83 to $84.35 \%$ of reads per library were mapped to annotated protein coding genes in the draft genome G. hirsutum, TM-1 [26]. A total of 61,263 genes were mapped in this Upland cotton genome for all four libraries (Additional file 1). Expressed genes were annotated with Arabidopsis Tair 10 homeolog genes. The percentages of genes having reads per kilo-base per million reads (RPKM) $>1$ were ranged from 71.61 to $79.22 \%$ per library. We selected an RPKM

Table 4 Summary of total raw and clean sequence tags

\begin{tabular}{|c|c|c|c|c|c|c|c|c|}
\hline \multirow{3}{*}{$\begin{array}{l}\text { Item } \\
\text { Time point } \\
\text { Replication }\end{array}$} & \multicolumn{4}{|l|}{ MD52ne } & \multicolumn{4}{|l|}{ MD90ne } \\
\hline & \multicolumn{2}{|l|}{15 DPA } & \multicolumn{2}{|l|}{20 DPA } & \multicolumn{2}{|l|}{15 DPA } & \multicolumn{2}{|l|}{ 20DPA } \\
\hline & Rep. 1 & Rep. 2 & Rep. 1 & Rep. 2 & Rep. 1 & Rep. 2 & Rep. 1 & Rep. 2 \\
\hline Total bases (million) & 3382.41 & 3247.35 & 3187.87 & 3382.75 & 3347.42 & 3313.08 & 3384.49 & 3096.28 \\
\hline Raw reads (million) & 33.49 & 32.15 & 31.56 & 33.49 & 33.14 & 32.80 & 33.51 & 30.66 \\
\hline GC\% & 44.31 & 44.21 & 44.69 & 45.32 & 44.28 & 44.48 & 45.00 & 45.03 \\
\hline Mapped reads (million) & 27.54 & 27.12 & 25.51 & 25.87 & 27.9 & 27.54 & 27.10 & 24.78 \\
\hline$\%$ Mapped reads & 82.23 & 84.35 & 80.83 & 77.25 & 84.19 & 83.96 & 80.87 & 80.82 \\
\hline Reads in genes (million) & 23.20 & 23.75 & 22.34 & 22.36 & 24.25 & 24.24 & 23.72 & 21.7 \\
\hline$\%$ reads in genes & 69.27 & 73.87 & 70.79 & 66.77 & 73.17 & 73.9 & 70.78 & 70.78 \\
\hline Total genes $>1 \mathrm{rpkm} \wedge$ & 46045 & 46263 & 48202 & 48532 & 47014 & 43868 & 47795 & 47267 \\
\hline Genes > 1 rpkm^ (\%) & 75.16 & 75.52 & 78.68 & 79.22 & 76.74 & 71.61 & 78.02 & 77.15 \\
\hline Genes $>0$ Reads & 47097 & 47319 & 49253 & 49600 & 48099 & 45007 & 48858 & 48390 \\
\hline Total genes >0 Reads (\%) & 76.88 & 77.24 & 80.40 & 80.96 & 78.51 & 73.47 & 79.75 & 78.99 \\
\hline
\end{tabular}


threshold of 1 to be consistent with prior work using this draft genome [26].

Expressed mapped genes were further evaluated between two NILs using RPKM values for the respective time points. The false discovery rate (FDR) was set at $5 \%$ to determine the threshold of $p$ value in multiple tests and analyses. To judge the significance of gene expression difference, adjusted $p \leq 0.05$ and the absolute value of $\log _{2}$ ratio $\geq 1$ was used as the threshold [27]. Of the 37,675 expressed genes, 4005 and 1080 unique genes were significantly differentially expressed at 15 and 20 DPA, respectively between the NILs (Fig. 4a, Additional files 2 and 3). Of the 4005 differentially expressed genes (DEGs), 3565 and 440 unique genes were expressed as up- and down-regulated in MD52ne at 15 DPA respectively, while out of 1080 genes, 774 and 306 DEGs were expressed up- and down-regulated in MD52ne at 20 DPA, respectively (Fig. 4b, Additional files 4, 5, 6 and 7).

\section{Annotation and gene ontology analyses of DEGs in MD52ne fibers}

GO enrichment analysis of the annotated DEGs by agriGo [28] showed that the DEGs involved in responses to stimuli and phytohormones, intracellular signaling, cellular metabolic process, cell wall modification, lipid localization, and carbohydrate metabolic process were commonly identified in developing MD52ne fibers at 15 and 20 DPA (Fig. 4c and Table 5). Numerous transcription factors regulated by growth stimulating phytohormones such as auxin, ethylene, and gibberellins (GA) were differentially expressed in developing MD52ne fibers (Table 5). Among them, 1-aminocyclopropane-1-carboxylic acid synthase 6 (ACC6) and 1-aminocyclopropane-1carboxylate oxidase 4 (ACO4), key enzymes for synthesizing ethylene stimulating fiber elongation [29, 30], and EIN3-binding F box protein 1 (EBF1) involved in ethylene signaling [31] were up-regulated at 15 and 20 DPA. GAST1 encoding for a GA promoting growth enzyme [32] was highly up-regulated in MD52ne. We also conducted GO enrichment analysis using DEGs at 15 and 20 DPA separately (Additional file 8).

Interestingly, multiple receptor-like kinases (RLKS) localized in plasma membranes were differentially expressed in the developing fibers of MD52ne as compared with MD90ne. Among the various classes of RLKs described in Fig. 5, the leucine rich repeat (LRR) RLKs containing three domains (LRR ligand binding motif, a transmembrane region and a kinase domain) were most frequently identified. The LRR RLKs have been recently suggested as a novel signaling pathway regulating plant cell wall integrity maintenance [33] and cellulose deposition in Arabidopsis [34, 35]. An LRR RLK (Gh_D08G0203) was one of the DEGs that were most highly up-regulated in developing MD52ne fibers
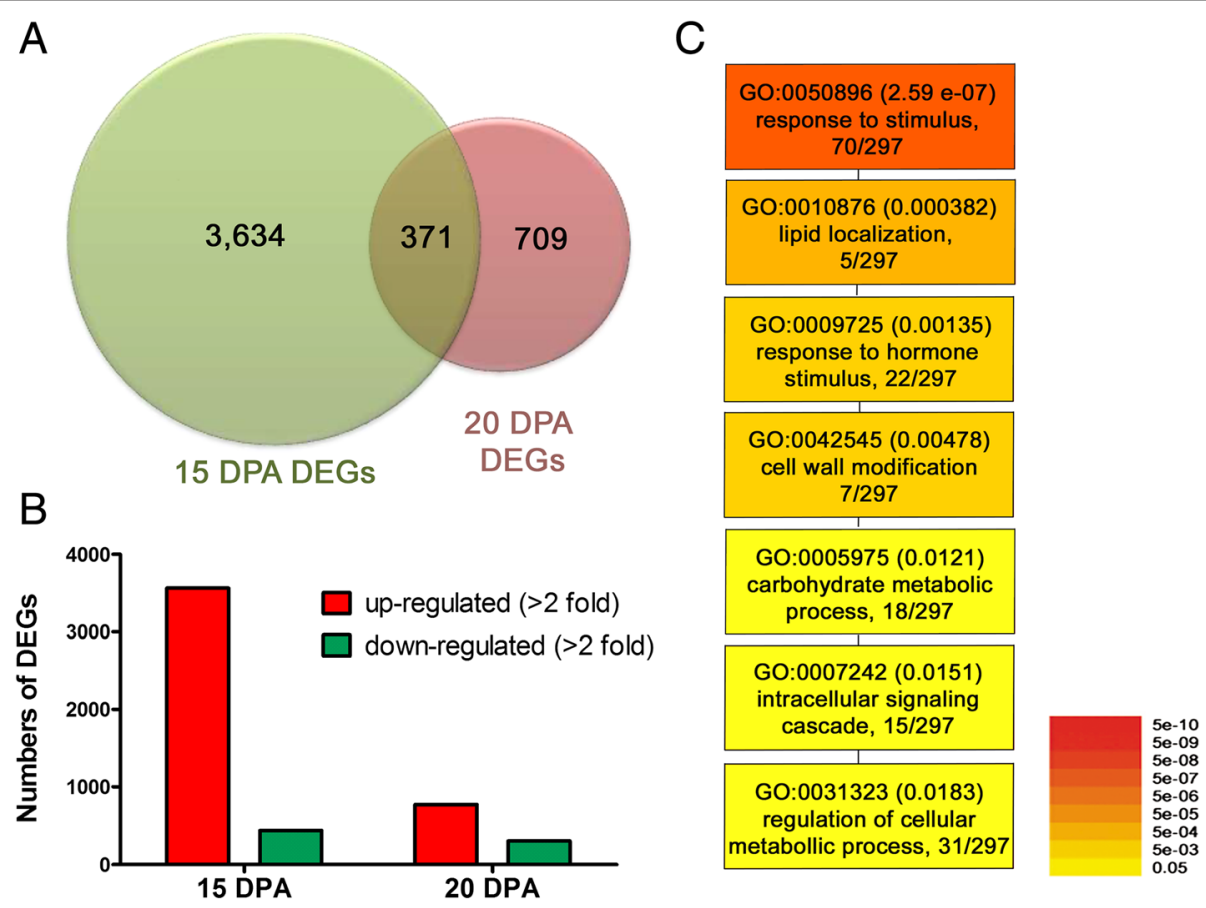

Fig. 4 Summary of RNA-seq analysis comparing MD52ne and MD90ne. a Venn diagrams representing differentially expressed genes (DEGs) at two developmental stages (15 and 20 DPA) in MD52ne and MD90ne fibers. b Comparisons of up- or down-regulated DEGs at 15 and 20 DPA in MD52ne fibers. c GO analysis. Singular enrichment analysis was used to identify GO categories that were commonly found at both 15 and 20 DPA developing fibers from MD52ne. The color and numbers adjacent to the GO identifier represent $p$-values 
Table 5 Annotation of genes differentially expressed in MD52ne at 15 and 20 DPA fiber

\begin{tabular}{|c|c|c|c|c|}
\hline \multirow[t]{2}{*}{ Gene ID } & \multirow[t]{2}{*}{ Tair 10 ID } & \multirow[t]{2}{*}{ Description } & \multicolumn{2}{|c|}{ Fold (MD52ne/MD90ne) } \\
\hline & & & 15 DPA & $20 \mathrm{DPA}$ \\
\hline \multicolumn{5}{|c|}{ Response to hormone stimulus } \\
\hline Gh_A08G0989 & AT4G11280 & 1-aminocyclopropane-1-carboxylic acid synthase 6 (ACC6) & 6.87 & 2.40 \\
\hline Gh_A07G0774 & AT1G05010 & 1-aminocyclopropane-1-carboxylate oxidase 4 (ACO4) & 6.35 & 4.28 \\
\hline Gh_A05G0085 & AT2G25490 & EIN3-binding $\mathrm{F}$ box protein 1 (EBF1) & 5.01 & 2.28 \\
\hline Gh_A09G1760 & AT4G17500 & Ethylene responsive element binding factor 1 & 2.45 & 2.60 \\
\hline Gh_A12G1554 & AT1G13260 & Related to ABI3NP1 1 & 4.21 & 4.76 \\
\hline Gh_D11G0427 & AT5G07580 & Integrase-type DNA-binding superfamily protein & 7.92 & 2.64 \\
\hline Gh_A11G2242 & AT5G43700 & AUX/IAA transcriptional regulator family protein & 10.19 & 7.99 \\
\hline Gh_A11G0443 & AT2G14960 & Auxin-responsive GH3 family protein & 2.90 & 0.26 \\
\hline Gh_A08G1509 & AT4G16780 & Homeobox protein 2 & 5.01 & 2.40 \\
\hline Gh_D08G2557 & AT5G57050 & Protein phosphatase $2 \mathrm{C}$ family protein & 3.56 & 2.12 \\
\hline Gh_D06G0657 & AT4G26080 & Protein phosphatase $2 \mathrm{C}$ family protein & 2.68 & 2.24 \\
\hline Gh_D09G0145 & AT5G15230 & GAST1 protein homolog 4 & 3.96 & 3.19 \\
\hline Gh_A06G0128 & AT5G25900 & GA requiring 3 & 0.42 & 0.44 \\
\hline Gh_D06G0417 & AT1G01720 & NAC transcriptional regulator & 2.72 & 3.23 \\
\hline Gh_D01G0514 & AT4G27410 & NAC transcriptional regulator & 2.58 & 2.09 \\
\hline \multicolumn{5}{|c|}{ Inter \& intra-cellular singaling cascade } \\
\hline Gh_D08G0203 & AT5G48940 & Leucine rich repeat receptor-like kinase (LRR RLK) & 376.83 & 701.62 \\
\hline Gh_D12G0208 & AT1G49740 & PLC-like phosphodiesterases superfamily protein & 38.86 & 6.53 \\
\hline Gh_A11G1809 & AT1G13680 & PLC-like phosphodiesterases superfamily protein & 5.13 & 4.88 \\
\hline Gh_D11G0427 & AT5G07580 & Integrase-type DNA-binding superfamily protein & 7.92 & 2.64 \\
\hline Gh_A05G2005 & AT5G12480 & Calmodulin-domain protein kinase 7 & 12.98 & 15.75 \\
\hline Gh_A07G0928 & AT5G59010 & Protein kinase protein with tetratricopeptide repeat domain & 4.75 & 2.48 \\
\hline Gh_A02G1701 & AT2G17220 & Protein kinase superfamily protein & 3.77 & 2.06 \\
\hline Gh_A03G0393 & AT5G59010 & Protein kinase protein with tetratricopeptide repeat & 3.11 & 2.71 \\
\hline Gh_D10G0338 & AT2G33170 & Leucine-rich repeat receptor-like kinase & 2.18 & 2.33 \\
\hline Gh_D05G1232 & AT1G11300 & Protein serine/threonine kinases & 0.41 & 2.94 \\
\hline Gh_D12G0893 & AT1G54610 & Protein kinase superfamily protein & 0.39 & 0.18 \\
\hline Gh_D01G1037 & AT4G20140 & Leucine-rich repeat transmembrane kinase & 0.34 & 0.30 \\
\hline Gh_A12G0190 & AT1G49820 & S-methyl-5-thioribose kinase & 0.20 & 0.27 \\
\hline Gh_A03G0234 & AT2G30500 & Kinase interacting (KIP1-like) family protein & 0.00 & 0.01 \\
\hline Gh_D12G2621 & AT3G22370 & Alternative oxidase $1 \mathrm{~A}$ & 0.03 & 0.17 \\
\hline \multicolumn{5}{|c|}{ Cell wall modification } \\
\hline Gh_D13G0786 & AT1G26770 & Expansin A10 & 3.72 & 2.14 \\
\hline Gh_A04G0707 & AT2G39700 & Expansin A4 & 3.67 & 4.21 \\
\hline Gh_A05G1180 & AT3G14310 & Pectin methylesterase 3 (PME3) & 3.41 & 6.70 \\
\hline Gh_A03G1432 & AT4G03210 & Xyloglucan endotransglucosylase/hydrolase 9 (XET9) & 2.56 & 1.03 \\
\hline Gh_A11G0768 & AT4G28850 & Xyloglucan endotransglucosylase/hydrolase 9 (XET26) & 3.97 & 1.34 \\
\hline Gh_D08G1309 & AT4G02280 & Sucrose synthase 3 (Sus3) & 4.70 & 1.00 \\
\hline Gh_A07G0665 & AT5G37180 & Sucrose synthase 5 (Sus5) & 13.97 & 0.94 \\
\hline Gh_D12G0298 & AT3G29810 & COBRA-like protein 2 precursor & 1.23 & 11.09 \\
\hline Gh_A06G0439 & AT3G12500 & Chitinase/ Chitinase-like (CHI/CTL) & 1.00 & 16.63 \\
\hline Gh_D06G0479 & AT3G12500 & Chitinase/ Chitinase-like (CHI/CTL) & 1.84 & 10.61 \\
\hline
\end{tabular}


Table 5 Annotation of genes differentially expressed in MD52ne at 15 and 20 DPA fiber (Continued)

\begin{tabular}{|c|c|c|c|c|}
\hline \multicolumn{5}{|c|}{ Lipid localization } \\
\hline Gh_A11G0232 & AT2G45180 & Lipid-transfer protein & 27.06 & 100.50 \\
\hline Gh_D07G1618 & AT5G48485 & Lipid-transfer protein & 3.54 & 2.78 \\
\hline Gh_A05G0073 & AT2G10940 & Lipid-transfer protein & 4.66 & 4.32 \\
\hline Gh_D13G1979 & AT4G30950 & Fatty acid desaturase 6 & 2.42 & 2.44 \\
\hline \multicolumn{5}{|c|}{ Transcriptional regulation of cellular metabolic process } \\
\hline Gh_D06G1433 & AT1G29160 & Dof-type zinc finger DNA-binding protein & 21.26 & 3.01 \\
\hline Gh_A13G1525 & AT1G22490 & bHLH DNA-binding protein & 10.53 & 7.80 \\
\hline Gh_D08G0923 & AT5G57660 & CONSTANS-like 5 & 4.64 & 2.46 \\
\hline Gh_D03G1189 & AT1G07530 & SCARECROW-like 14 & 2.65 & 2.71 \\
\hline Gh_A09G0414 & AT5G52510 & SCARECROW-like 8 & 2.07 & 2.22 \\
\hline Gh_D06G1966 & AT1G80840 & WRKY DNA-binding protein 40 & 5.40 & 3.25 \\
\hline Gh_D12G1243 & AT1G13960 & WRKY DNA-binding protein 4 & 2.35 & 3.28 \\
\hline Gh_D09G1456 & AT5G56270 & WRKY DNA-binding protein 2 & 2.02 & 2.29 \\
\hline Gh_A12G1763 & AT1G57820 & Zinc finger (C3HC4-type RING finger) protein & 4.32 & 6.50 \\
\hline Gh_A01G1274 & AT5G50570 & Squamosa promoter-binding transcription factor & 4.67 & 3.05 \\
\hline Gh_A11G2041 & AT1G69560 & MYB protein 105 & 3.66 & 2.51 \\
\hline Gh_A12G1244 & AT4G21440 & MYB-like 102 & 0.21 & 0.46 \\
\hline Gh_A04G0781 & AT2G38250 & Homeodomain-like superfamily protein & 3.67 & 2.29 \\
\hline Gh_A08G1509 & AT4G16780 & Homeobox protein 2 & 5.01 & 2.40 \\
\hline Gh_A06G0833 & AT4G32880 & Homeobox gene 8 & 2.57 & 2.12 \\
\hline Gh_D08G2178 & AT1G09250 & Basic helix-loop-helix (bHLH) DNA-binding protein & 5.23 & 2.38 \\
\hline Gh_D12G2057 & AT5G46910 & Transcription factor jumonji (jmj) zinc finger (C5HC2 type) & 2.18 & 2.11 \\
\hline Gh_D11G0427 & AT5G07580 & Integrase-type DNA-binding superfamily protein & 7.92 & 2.64 \\
\hline Gh_A10G0516 & AT3G13890 & MYB protein 26 & 9.28 & 0.87 \\
\hline Gh_A09G1074 & AT5G12870 & MYB protein 46 & 7.81 & 1.03 \\
\hline
\end{tabular}

at both 15 DPA (377 fold) and 20 DPA (702 fold) (Additional file 9).

Cotton genes encoding expansins [36, 37], pectin methylesterase (PME) [38], and xyloglucan endotransglucosylase/hydrolases (XET) [39] required for expanding the PCW during the fiber elongation stage were up-regulated in developing MD52ne fibers. Five expansins (Gh_D13G0786, Gh_A11G2917, Gh_A04G0707, Gh_A12G1619, and Gh_A12G1619), three PMEs (Gh_A05G1180, Gh_D06G0865, and Gh_D07G0145), and five XETs (Gh_A03G1432, Gh_D02G1891, Gh_D05G0764, Gh_A11G0768, and Gh_D13G0290) were highly expressed in MD52ne fibers (Table 5). Four Sus (Gh_A07G0665, Gh_A08G1031, Gh_D08G1309, and Gh_D11G0438) and multiple lipid transfer proteins involved in fiber development $[29,40,41]$ were co-expressed at actively elongating MD52ne fibers.

COBRA-like protein 2, whose sequence is similar to Arabidopsis COBRA-like proteins involved in cellulose microfibrils orientation in Arabidopsis [42], and Chitinase/Chitinase-like (CHI/CTL), similar to an Arabidopsis
CTL2 responsible for crystalline cellulose content in Arabidopsis [43], were specifically up-regulated in the SCW stage (20 DPA) of MD52ne. Several transcription factors including NAC (No Apical Meristem), bHLH (basic helix-loop-helix), COL5 (CONSTANS-like 5), WRKY, and zinc finger family protein were differentially expressed in developing MD52ne fibers at both 15 and 20 DPA, whereas MYB 26 and 46 transcription factors orthologous to Arabidopsis MYB26 (AT3G13890) and 46 (AT5G12870) involved in SCW biosynthesis [44, 45] were specifically up-regulated in developing MD52ne fibers at 15 DPA (Table 5).

\section{Validation of DEGs in MD52ne fibers}

The expression patterns of the DEGs identified from RNA-seq data of the two NILs were validated with real time quantitative PCR (RT-qPCR) analysis. Based on GO enrichment analysis, 32 DEGs showing different expression pattern in the RNA-seq were selected for RT-qPCR using RNAs from developing fibers at 10, 15, 20 and 24 DPA. Comparative transcript levels of the all 32 DEGs 


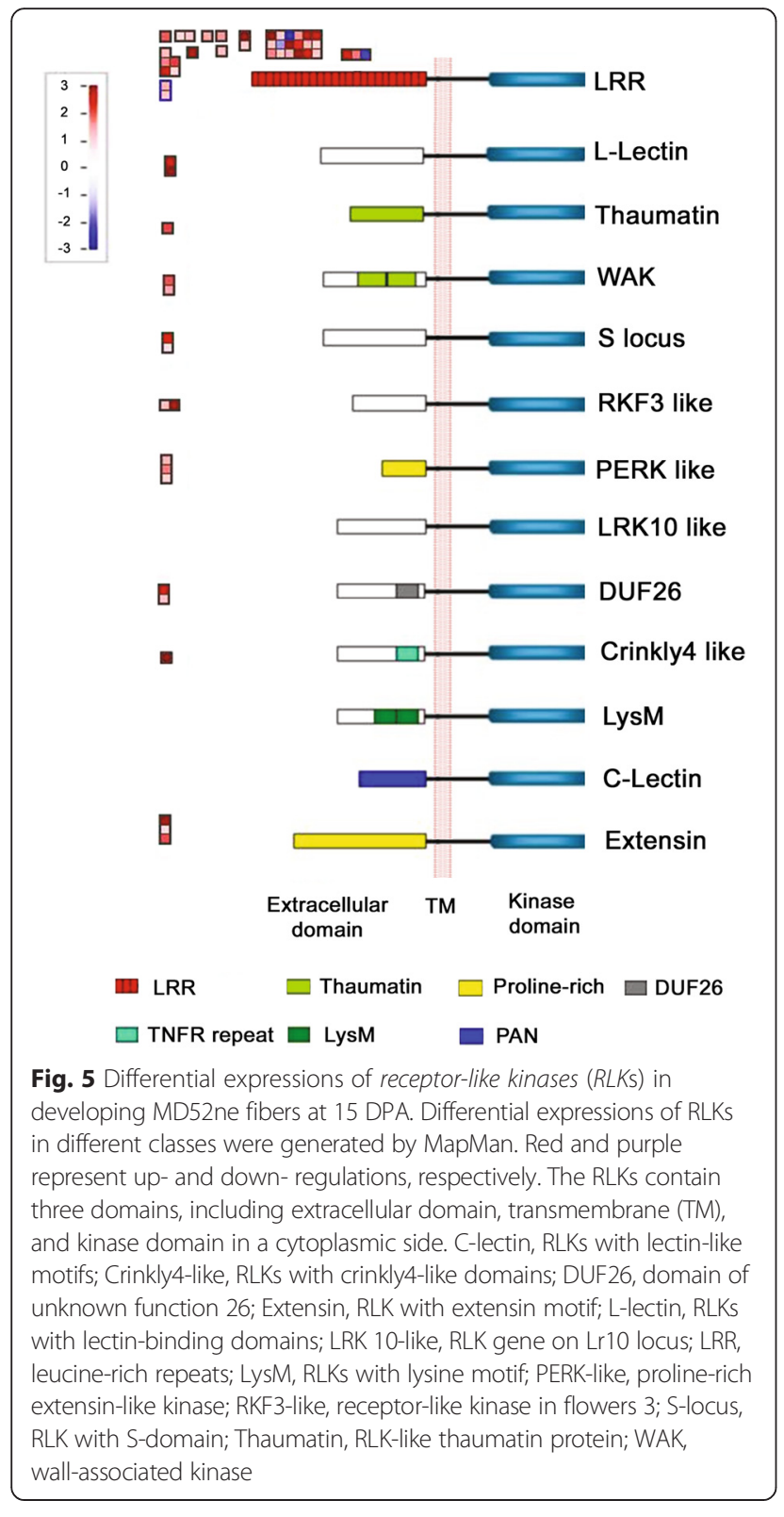

obtained by both RT-qPCR and RNA-seq were presented in Additional file 10, and the RT-qPCR results from the 9 critical DEGs were shown in Fig. 6. The identical expression patterns of all the tested DEGs between RT-qPCR and RNA-seq indicated the reliability of both sequencing and the DEG filtering process.

$A C O 4$ and EBF1, which are both involved in the ethylene signaling pathway $[29,30]$, were expressed more in MD52ne than in MD90ne at all stages in PCW biosynthesis (10 DPA), transition (15 DPA), and SCW biosynthesis (20 and 24 DPA) (Fig. 6). The most significant upregulation in all developmental stages was found in the $L R R R L K$ that is a new signaling pathway of cell wall integrity [33]. A transcription factor showing sequence similarity to Arabidopsis $M Y B \quad 46$ regulating biosynthesis of cellulose, hemicelluloses, and lignin $[44,46]$ was expressed more abundantly in MD52ne than in MD90ne specifically at the transition stage (15 DPA) between the PCW and SCW biosyntheses (Fig. 6). Consistently, cellulose synthase catalytic subunit A4 (CesA4) which is involved in the SCW biosynthesis of cotton fiber [47] showed an identical expression pattern to the MYB46 transcription factor. The expression pattern of a zinc finger protein COL5 involved in cellular metabolic process was also highly abundant in MD52ne at all tested DPAs. A CHI/CHI (Gh_D06G0479) responsible for crystalline cellulose content in other plants $[43,48]$ was highly up-regulated in MD52ne as compared to MD90ne. During the active SCW biosynthesis stage (20-24 DPA), the transcript levels of $\mathrm{CHI} / \mathrm{CHI}$ were 30 fold higher in MD52ne fibers than in MD90ne fibers. An FAD-linked oxidase and a ribosomal L18e/L15 protein (L18e/L15) were highly up-regulated in MD52ne over MD90ne in all developmental stages.

\section{DEGs at the QTL regions for bundle strength and fiber length}

We previously identified stable quantitative trait loci (QTLs) associated with BFS and fiber length (UHML) using an $F_{2}$ population derived from a cross between MD52ne and MD90ne [49]. The QTLs associated with BFS and UHML were linked with simple sequence repeat markers, BNL4034 and GH454 located on chromosome 3 (A03) and chromosome 24 (D08), respectively. We aligned the QTL regions along with the DEGs with the physical map of the G. hirsutum TM-1 genome [26, 50]. A total of 75 genes were differentially expressed either at 15 and 20 DPA in the QTL regions. Of the 75 DEGs, 17 genes (9 DEGs in the BFS QTL and 8 DEGs in the UHML QTL) up-regulated more than 2 fold in MD52ne were involved in cell wall modification based on GO analysis (Additional file 11). Three $L R R R L K s$, two NAC transcription factors, COL5, MADS-box transcription factor, and WRKY transcription factor involved in phytohormonal and RLK signaling pathways were located on the QTL regions (Fig. 7). Trehalose-phosphatse / synthase 9, XET9, Sus3, and germin like protein involved in cell wall biosynthesis or wall carbohydrate metabolisms were also found near the QTL regions. Two ribosomal L18e/L15 proteins were also located on chromosome A03 QTL location. Among them, the ribosomal L18e/L15 protein (Gh_D02G1619) at the A03 QTL and the LRR RLK (Gh_D08G0203) located near the D08 QTL were the DEGs that were mostly upregulated at all developmental stages (Fig. 6).

\section{Discussion}

Greater force is required for breaking individual fibers of MD52ne than its NIL, MD90ne

The BFS value has been broadly used to evaluate fiber strength which is the breaking force of a fiber bundle. In 

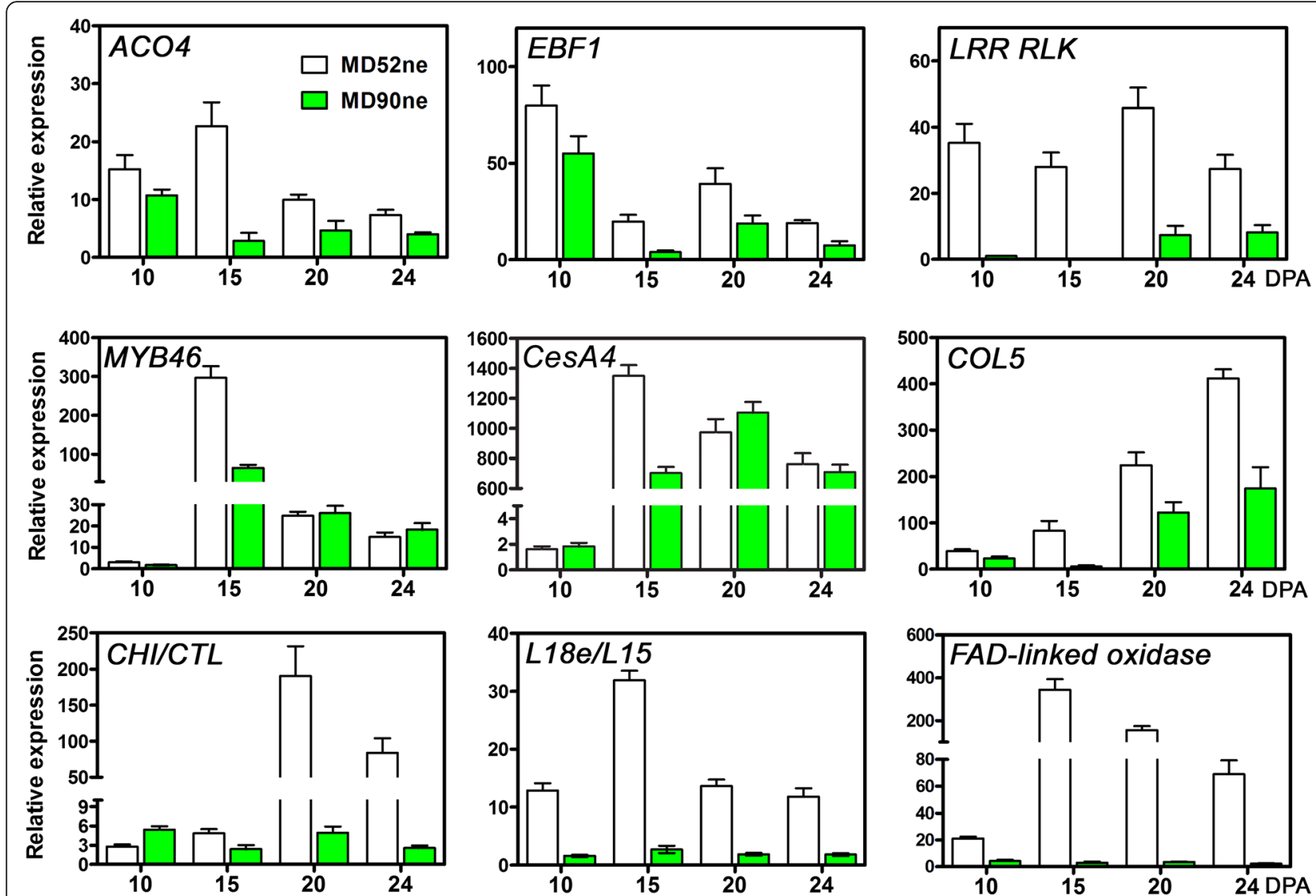

Fig. 6 RT-qPCR validation of selected genes related to cell wall activity during fiber development. Three biological replications and three technical replications at four fiber development stages (10, 15, and 24 DPA) were used for RT-qPCR analyses. ACO4, 1-aminocyclopropane-1-carboxylate oxidase 4, or ethylene forming enzyme (Gh_A07G0774); EBF1, EIN3-binding F box protein 1 (Gh_A05G0085); LRR RLK, Leucine rich repeat receptor-like kinase (Gh_D08G0203); MBY46 transcription factor (Gh_A09G1074); CesA4, Cellulose synthase catalytic subunit A4 (Gh_A08G0421); COL5,CONSTANS like 5 (Gh_D08G0923); CHI/CTL, Basic-Chitinase/Chitinase-like (Gh_D06G0439); L18e/L15, Ribosomal L18e/L15 protein (Gh_D02G1619); FAD-linked oxidase (Gh_D02G1214)

addition to the breaking force, the BFS is also affected by variable fiber properties like length, fineness, maturity, and MIC involved in fiber-to-fiber interactions. The correlation analysis of fiber properties from the 384 $\mathrm{F}_{2}$ progenies from a cross between MD52ne and MD90ne showed that the BFS variation of the NILs was mainly determined by the breaking force with low effects from variable fiber properties related to fiber-to fiber interactions (Table 2). The fiber length showing 5-6 \% differences between the NILs contributed a slight influence $(12.9 \%)$ on the BFS variances between the NILs, whereas the fiber thickness related properties having insignificant variations between the NILs had almost no effect on the BFS variances of the NILs (Tables 1 and 2). Since the BFS variance of the NILs is mainly driven by breaking force, the ratio of the bundle strength between the NILs was expected to be similar to the ratio of the individual fiber strength that is not affected by fiber-to fiber interactions. As predicted, the strengths of bundle (24 \%) and individual fibers (22 \%) from the MD52 were similarly higher than those from the MD90ne (Tables 1 and 3). As results, we concluded that the superior breaking force mainly contributed to the high bundle strength of the MD52ne fibers with a minor contribution from longer fiber. Compared with the cotton CSIL lines which have been used for studying fiber strength despite great potential effects from fiber length and thickness related properties due to their substantial variances among the CSILs $[18,19]$, the MD52ne and MD90ne are more ideal cotton NILs for dissecting molecular mechanisms of intrinsic fiber strength since their BFS variance was mainly affected by the breaking force but minimally regulated by other fiber properties involved in fiber-to fiber interactions within a fiber bundle.

Based on the fiber property analyses of the developing fibers whose crystallinity increased rapidly from 15 to 17 DPA (Fig. 3b), we determined that the transition from PCW to SCW biosynthesis stages began at approximately 15 DPA. Thus, we compared the transcript abundance in developing MD52ne and MD90ne fibers at two 


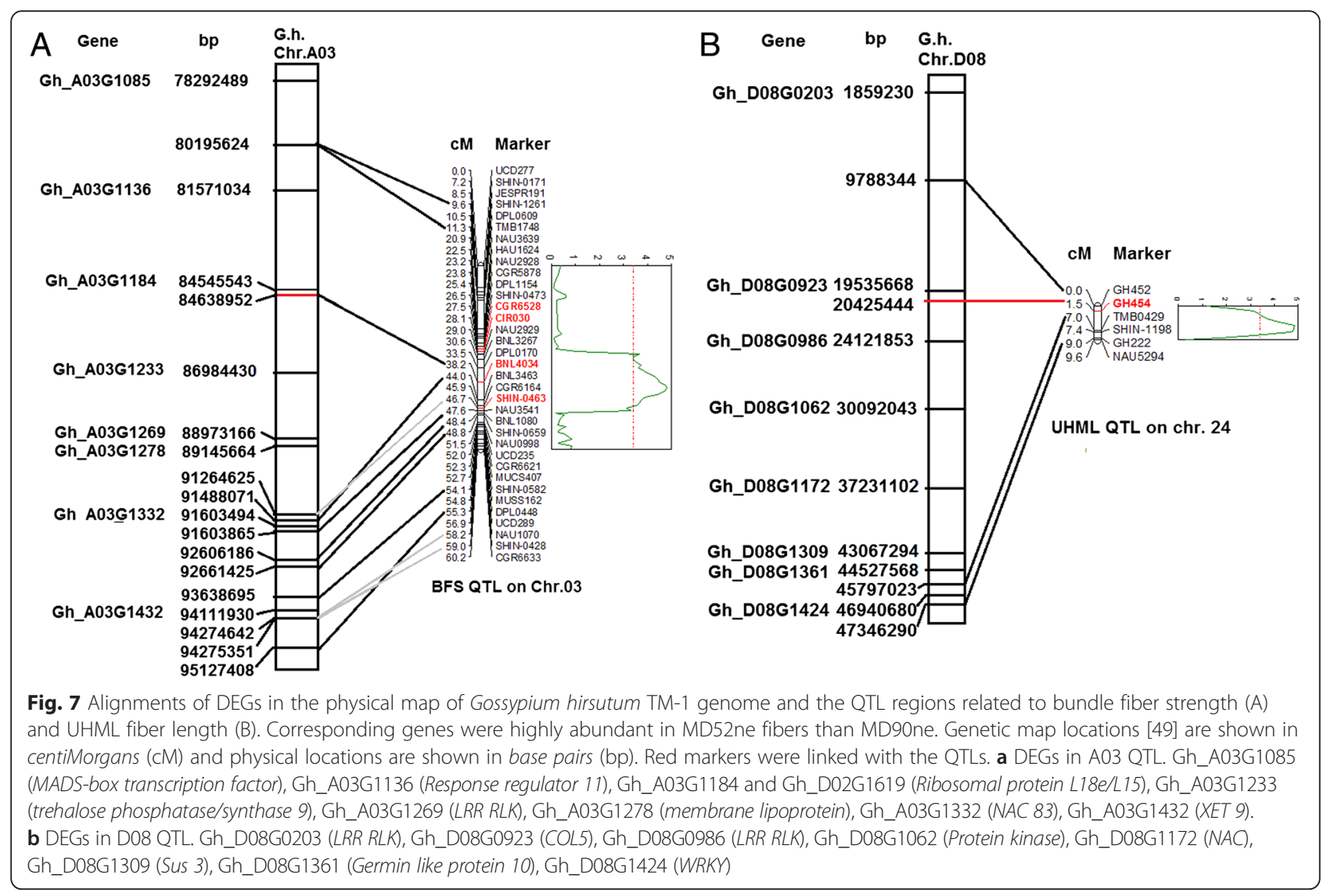

different time points: 15 DPA in which actively elongating fibers mainly consisted of PCW with low crystallinity (MD52ne, $18.1 \%$; MD90ne, $20.9 \%$ ), and 20 DPA in which SCW thickening fibers were composed of both PCW and SCW with high crystallinity (MD52ne, 38.4 \%; MD90ne, $41.5 \%$ ). In the developing fibers at the transition stage, a new cell wall layer named as winding layer is deposited [51]. Based on the observation of high fiber strength in developing fibers (21 DPA) composed of a winding layer with minimum SCW [8], the winding layer has been speculated as a potential source of fiber strength [17, 52]. To determine if and how the winding layer contributes to the strength of bundle and individual fibers, further comprehensive studies may be necessary since single fiber strength of developing fibers was previously measured by an Instron tensile tester that was a prototype for measuring individual fiber strength [8] and caused high variability and inconsistency [53]. In our studies, high BFS values of the NILs were also detected at the transition stage (20 DPA) of the NILs (Fig. 3e). The BFS of developing MD52ne fibers $(21.73 \mathrm{~g} /$ tex, $20 \mathrm{DPA})$ was significantly ( $p$ value, 0.0027) higher than that of developing MD90ne fibers (17.52 g/tex, 20 DPA).
Ethylene and its networking phytohormonal pathways may be involved in superior fiber length development in MD52ne

Comparative transcriptome analyses showed that transcripts related to ethylene and its networking auxin and GA signaling pathways for promoting fiber elongation were highly abundant in MD52ne (Table 5 and Fig. 4c). Ethylene gas is known as a major phytohormone stimulating fiber elongation [54]. Up-regulations of ethylene synthesizing genes like ACC and ACO as well as ethylene signaling gene like EBF are critical for active fiber elongation [29-31]. Consistent with the prior findings, $A C C 6, A C O 4, E B F 1$, and ERF1 involved in ethylene biosynthesis and signaling pathway required for fiber elongation were highly expressed in elongating MD52ne fibers whose length (UHML) was longer than its NIL, MD90ne (Table 5 and Fig. 6). In addition, transcripts (AUX/IAA, auxin-responsive GH3 protein, and GAST1) involved in auxin and GA were required for differentiating and elongating fibers $[41,55]$. For promoting fiber elongation, multiple expansins involved in loosening the cell walls and lipid transfer proteins involved in fiber elongating [29] were also up-regulated in the MD52ne fibers (Table 5 and Fig. 8). A large set of genes (XET, PME, and Sus) involved in xyloglucan and pectin biosynthesis 


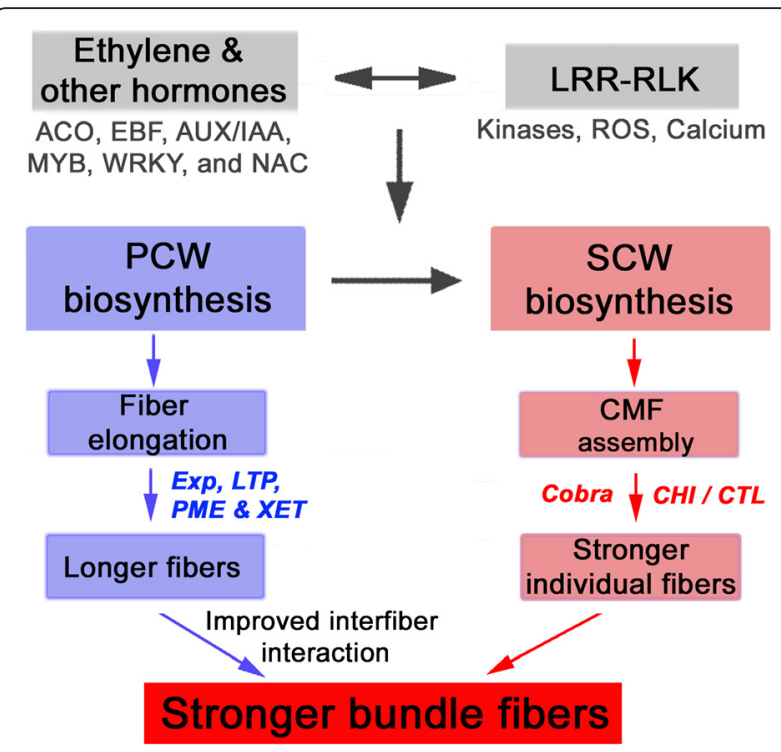

Fig. 8 Proposed model for the mechanism responsible for high fiber bundle strength and individual fiber breaking force in MD52ne. Abbreviated names included in this model are: LRR RLK, Leucine rich repeat receptor-like kinase; $\mathrm{PCW}$, primary cell wall; SCW, secondary cell wall; CME, cellulose micrfibrils; ACO, 1-aminocyclopropane-1carboxylate oxidase; EBF, EIN3-binding F box protein; AUX/IAA, AUX/ IAA transcription factor; MYB, MYB transcription factor: WRKY, WRKY transcription factor; NAC, NAC transcription factor; Exp, Expensin; LTP, Lipid transfer protein; PME, Pectin methylesterase; XET, Xyloglucan endotransglucosylase; Cobra, Cobra like protein; $\mathrm{CHI} / \mathrm{CTL}$, Chitinase/Chitinase-like protein; ROS, Reactive oxygen species

and carbohydrate metabolism requiring cotton fiber elongation [56] was also enriched in the elongating MD52ne fibers (Tables 5 and 6). A XET (Gh_A03G1432) and a Sus (Gh_D08G1309) were linked with the QTLs associated with BFS and UHML (Fig. 7).

\section{Receptor-like kinase signaling pathway regulating} cellulose deposition and maintaining cell wall integrity may be involved in superior fiber strength development in MD52ne

In addition to phytohormone and transcriptional networks controlling plant growth and development, receptor-like kinases (RLKs) have been found as novel regulators for both plant development and stress responses $[33,57]$. Among the various RLK classes described in Fig. 5, RLKs containing a leucine-rich repeat (LRR) were most frequently identified from developing MD52ne fibers. Three LRR RLKs were also found in the two QTLs associated with BFS and length (Table 5, Figures 6 and 7). In Arabidopsis elongating root tips and seeds, two LRR RLKs named FEI 1 and 2 have been reported to play a role in cellulose deposition in Arabidopsis elongating root tips [34] and seed coat [35]. ACC (1-aminocyclopropane-1-carboxylic acid) that is essential for ethylene signaling has been suggested to be a signaling molecule for FEI 1 and 2 [58], so both ethylene and LRR RLK signaling are most likely involved in cellulose deposition in elongating tissues (Fig. 8). Other LRR RLKs whose ligands are unknown are reported as a regulator of the SCW formation in Arabidopsis [59] and popular trees [60]. A cotton LRR RLK named GhRLK1 located in the plasma membrane was reported to be induced during active SCW synthesis stage [61]. Therefore, LRR RLK signaling pathways might be involved in mediating a coordination of cell elongation and SCW biosynthesis during cotton fiber development as suggested in other plants $[57,58]$.

\section{Temporal regulation of the genes involved in crystalline cellulose assembly at secondary wall biosynthesis stage of MD52ne fibers}

Three genes (Gh_D12G0298, Gh_A13G0320, and Gh_D13G0359) encoding COBRA-like protein were specifically up-regulated during the SCW biosynthesis stage in MD52ne fibers (Table 5). COBRA-like protein 2, a member of the GPI-anchored COBRA-like family, has been recently identified to play a role in crystalline cellulose deposition in Arabidopsis seed coat [62]. When a COBRA-like protein was deficient in brittle culm1 rice mutant [63] or brittle stalk2 maize mutant [64], mechanical strength of the stems was reduced. A COBRA-like protein interacting with cellulose modulates cellulose assembly in rice [42]. Therefore, the three COBRA-like proteins up-regulated at the SCW stage of MD52ne fibers can be candidate genes that contribute to the superior strength of MD52ne (Fig. 8).

Four CHI/CTL genes (Gh_D06G0479, Gh_A06G0439, Gh_A10G1271, and Gh_D09G2016) specifically upregulated in the SCW stage of MD52ne fibers were similar to the sequences of Chitinase $(\mathrm{CHI})$ and Chitinase like protein $(C T L)$ in other plants. CTL2 is responsible for crystalline cellulose content in Arabidopsis [43], whereas $\mathrm{CHI}$ is a pathogenesis-related gene responding to biotic stress in rice and maize $[65,66]$. Thus, the real function of $C H I / C T L$ genes identified from MD52ne remains to be determined.

\section{Temporal regulation of genes at transition stage of MD52ne fibers}

We previously reported temporal up-regulation of SCW biosynthesis-related genes at the transition from the PCW to SCW stage in the MD52ne fibers based on the transcriptome profiles performed with the first generation of cotton oligonucleotide microarray [16, 17]. The present transcriptome analyses performed with RNA-seq and G. hirsutum genome sequence identified many more DEGs in the MD52ne fibers (Additional file 12) than the previous microarray analysis [17]. The RNA-seq analysis also showed that some SCW biosynthesis-related genes such as MYB26, MYB46, and CesA4 [44, 45, 47] were 
up-regulated specifically at 15 DPA (Table 5 and Fig. 6). In contrast, other 26 CesAs identified by the RNA-seq did not show temporal up-regulation at the transition stage. For further analysis of the temporal regulation of SCW biosynthesis-related genes, we retrieved 181 Arabidopsis genes from PlaNet [67] that are temporally and spatially co-expressed during SCW biosynthesis in Arabidopsis. Among the MD52ne genes ortholgous to the 181 Arabidopsis SCW-related genes, 64 genes (35.4\%) showed temporal up-regulation in 15 DPA developing MD52 fibers (Additional file 13), whereas others showed no temporal up-regulation. In addition to the limited numbers of up-regulated SCW genes in the MD52ne over MD90ne, the identical levels of the crystalinity between the NILs (Fig. 2B) might imply that the genes related to SCW cellulose biosynthesis were less involved in the superior fiber strength of the MD52ne than the genes related to SCW cellulose assembly and wall integrity.

\section{Conclusions}

The demand of high fiber strength has been increased dramatically with the advent of modern high speed spinning technology for producing yarn. Cotton researchers have tried to improve this trait in G. hirsutum genetic backgrounds. MD52ne was proven to have higher fiber strength than its NIL MD90ne. This study was conducted to unveil the molecular mechanisms behind the formation of superior individual fiber strength, which is correlated with yarn strength, in MD52ne using RNA-seq technology. The bundle strength of the MD52ne fibers predominantly depends on individual fiber strength combined with fiber length. Comparative transcriptome analyses of the NILs suggested that the superior strength of MD52ne fibers was potentially related to two signaling pathways (Fig. 8): one is ethylene and its interconnected phytohormonal pathways involved in fiber elongation and interfiber interactions, and the other is RLKs signaling pathways involved in regulating cell wall integrity and potentially mediating a coordination of cell elongation and SCW biosynthesis. Several secondary cell wall biogenesis related genes and transcription factors such as COBRA-like protein, CHI/CTL, NAC, WRKY, COL5, Zinc finger family protein and MYB were up-regulated in MD52ne developing fibers. The superior BFS of MD52ne fibers might be the result of high individual fiber strength with a minor contribution from longer fiber length. The longer fibers may increase the fiber-to-fiber interactions and are likely the result of differential regulation of some PCW related genes. The improved individual fiber strength of MD52ne is likely related to pathways regulating cell wall integrity.

\section{Methods}

\section{Plant material}

The cotton NILs MD52ne and MD90ne were bred and provided by Dr. William Meredith of USDA-ARS-SEA (Additional file 14) [14, 15]. The two NILs were grown at two different fields for two growing seasons and at a greenhouse for one growing season. To compare fiber physical properties between the two NILs and determine variations in each NIL plant, ten individual plants of each NIL were grown at a field located in Stoneville, MS in 2012. The soil type in Stoneville is Bosket fine sandy loam. The mature fibers were collected from each plant of each NIL. To perform comparative transcriptome analyses, three biological replications (approximately 40 cotton bolls per replication) at each developmental time point $(10,13,15,17,20,24,28,33,37,44$, and $48 \mathrm{DPA})$ were collected from 50 plants of the two NILs grown at a field located in New Orleans, LA in 2013. The soil type in New Orleans was Aquent dredged over alluvium in an elevated location to provide adequate drainage. Fiber samples from $384 \quad F_{2}$ progeny plants derived from crosses between MD90ne and MD52ne were collected at Stoneville, MS, USA in 2012 as described in Islam et al. [49]. All naturally-open bolls were manually harvested from each of the $F_{2}$ plants. Developing fibers (10-37 DPA) were manually collected from ovules and mature fibers (44 DPA) were ginned using a laboratory roller gin. The collected fibers were frozen immediately with liquid nitrogen for RNA extraction or dried in $40{ }^{\circ} \mathrm{C}$ incubator for physical property analyses. To extract additional RNAs that were used for verification of the transcriptome results, three biological replications of cotton fibers at each developmental time point (10-44 DPA) were collected from the two NILs grown in 5 gal pots with Metro-Mix 360 in a greenhouse located in New Orleans, LA. Throughout all processes from planting, tagging, harvesting, and ginning, the two NILs grown side by side were equivalently treated.

\section{Fiber property measurements}

For measurements of fiber properties from cotton fibers, fibers were pre-equilibrated with $65 \pm 2 \%$ humidity at $21 \pm 1{ }^{\circ} \mathrm{C}$ for $48 \mathrm{~h}$. All fiber properties were obtained from instruments that were properly calibrated according to the manufacturers' instructions and standard cotton fibers were obtained from USDA-AMS.

BFS (g/tex), UHML (inch), FE (\%), and MIC values of the mature fibers from the two NILs were obtained from five replicates measured by HVI (USTER Technologies Inc., Knoxville, TN). To determine BFS from developing fibers, a stelometer (SDL Atlas, Stockport, England) was used with three replicates of fiber samples. BFS is given as tenacity, expressed as kilonewton meters per kilogram, and is the force required to break a bundle of 
fibers of a specific gravimetric linear density. For measuring the breaking force of individual fibers, Favimat (Textechno, Mönchengladbach, Germany) was used with 303 individual fibers and a $13 \mathrm{~mm}$ length gauge, according to Delhom et al. [68].

AFIS maturity ratio and fineness were measured using Uster $^{\circ}$ AFIS-Pro (USTER Technologies Inc., Knoxville, TN). The average AFIS fiber data were obtained from five replicates with 5000 fibers per replicate.

For measuring the gravimetric fineness (mtex, $\mathrm{mg} \mathrm{km}^{-1}$ ) of the fibers, three hundred fibers were combed, cut at the top and bottom to leave them $15 \mathrm{~mm}$ long, and measured by a microbalance [69]. Average gravimetric fineness was calculated from the three measurements.

Circularity $(\theta)$ representing the degree of fiber cell wall thickness was directly measured from light microscopic images from cross-sectioned fibers [70]. Average cell wall area (A), excluding lumen and perimeter $(\mathrm{P})$ of the fiber cross sections, was measured from 300 crosssections using the image analysis software according to $\mathrm{Xu}$ and Huang [71]. The obtained circularities from the equation, $\theta=4 \pi \mathrm{A} / \mathrm{P}^{2}[72]$ were converted to maturity ratio (MR) using the equation, $\mathrm{MR}=\theta / 0.577$ [73].

\section{ATR-FTIR spectral collection and data analysis}

All spectra were collected with an FTS 3000MX FTIR spectrometer (Varian Instruments, Randolph, MA) equipped with a ceramic source, $\mathrm{KBr}$ beam splitter, and deuterated triglycine sulfate (DTGS) detector. The ATR sampling device utilized a DuraSamplIR single-pass diamond-coated internal reflection accessory (Smiths Detection, Danbury, CT), and a consistent contact pressure was applied by way of a stainless steel rod and an electronic load display. At least six measurements at different locations for individual samples were collected over the range of $4000-600 \mathrm{~cm}^{-1}$ at $4 \mathrm{~cm}^{-1}$ and $16 \mathrm{co}-$ added scans. All spectra were given in absorbance units and no ATR correction was applied. Following the import to GRAMS IQ application in Grams/AI (Version 9.1, Thermo Fisher Scientific, Waltham, MA), the spectra were smoothed with a Savitzky-Golay function ( polynomial $=2$ and points $=11$ ). Then, the spectral set was loaded into Microsoft Excel 2000 to assess cotton crystallinity and maturity by using a previously proposed algorithm analysis $[23,24]$. In the original concept of assessing cellulose maturity $\left(\mathrm{M}_{\mathrm{IR}}\right)$ and crystallinity index $\left(\mathrm{CI}_{\mathrm{IR}}\right)$ from IR measurement [22-24], the key wavelengths was identified and then two algorithms $\left(R_{1}\right.$ and $R_{2}$ ) were developed to estimate the degree of cotton cellulose $M_{I R}$ and $C_{I R}$ by representing the $R_{1}$ and $R_{2}$ values. Both mean value and standard deviation for each fiber sample were used for the comparison between two types of varieties.
RNA extraction, library preparation and sequencing Total RNA was extracted from the developing cotton fibers $\left(10,15,20\right.$ and 24 DPA) using the Sigma Spectrum ${ }^{\mathrm{Ts}}$ Plant Total RNA Kit (Sigma-Aldrich, St. Louis, MO) with DNase1 digestion according to the manufacturer's protocol. The quality and quantity of total RNA were determined using a NanoDrop 2000 spectrophotometer (NanoDrop Technologies Inc., Wilmington, DE) and an Agilent Bioanalyzer 2100 (Agilent Technologies Inc., Santa Clara, CA). The RNA samples of two biological replications at two different developmental stages (15 and $20 \mathrm{DPA}$ ) from both NIL fibers were sent to Data2Bio LLC (Ames, Iowa) for library preparation and subsequent paired-end Illumina mRNA sequencing according to the methods that were previously described [74].

\section{RNA-seq data processing}

The raw RNA-seq reads were trimmed with SICKLE (https://github.com/najoshi/sickle) using a quality score cutoff of 20 . Then, the RNA-seq reads were aligned to the Gossypium hirsutum draft genome [26] with the GSNAP software program [75]. Reads mapped to each annotated gene were counted with Bedtools software [76].

\section{Identification of differentially expressed genes}

Differential gene expression was calculated by the negative binomial method of the EdgeR software using the tagwise estimation of dispersion [77]. RPKM was used to estimate gene expression levels as calculated: $\mathrm{RPKM}=\left[10^{9} / N L\right] \mathrm{C}$, where $\mathrm{C}$ stands for the number of reads that could map to the target unigene, $\mathrm{N}$ represents the number of reads that could map to at least one unigene, and $\mathrm{L}$ refers to the length of the target unigene. The accuracy of the test result was corrected by FDR. In this study, FDR $<0.05$ and the absolute value of the $\log _{2}$ ratio (in which 'ratio' refers to the fold change in expression of the target unigene in among libraries) were used to select DEGs.

\section{Validation of RNA-seq results with RT-qPCR}

The experimental procedures and data analysis related to RT-qPCR were performed according to the Minimum Information for Publication of Quantitative Real-Time PCR Experiments guidelines [70]. Four fiber development stages $(10,15,20$ and 24 DPA) were used for RT-qPCR analyses for validating the RNA-seq result of selected genes. The detailed description of cDNA preparation, $\mathrm{qPCR}$, and calculations were previously reported [20]. Specific primer pairs were designed from 32 DEGs for validation of the RNA-seq. The endogenous reference genes used in the RTqPCR reactions were the $18 \mathrm{~S}$ rRNA (U42827), and $\alpha$ tubulin 4 (AF106570). The reference and target gene primer sequences are shown in Additional file 15. Three 
biological replications and three technical replications for each time-point were used for RT-qPCR.

\section{Gene annotation analyses}

RNA-seq data obtained from 15 and 20 DPA fibers of two NILs were first subjected to Venn analysis utilizing BioVenn [78] to determine which DEGs were common between two time-points. To assist in the identification of biological processes represented in the data, GO enrichment analysis was performed using the agriGO Singular Enrichment Analysis [28]. The statistical test method used was the Fisher's exact test (significance level 0.05). For metabolic analysis, MapMan software [79] was used to identify and illustrate metabolic overview of cell wall related molecules.

\section{Ethics approval and consent to participate}

Not applicable.

\section{Availability of supporting data}

All supporting data can be found within the manuscript and its additional files. The biological sequences were deposited in the Sequence Read Archive (SRA), National Center for Biotechnology Information (NCBI) under the accession numbers SRS843151, SRS843159, SRS843160, and SRS843163.

\section{Additional files}

Additional file 1: Name of mapped gene in Gossypium hirsutum (TM-1) and their annotation with Arabidopsis homeolog genes. This table contains 61,263 allotetraploid cotton genes that aligned to Gossypium hirsutum (TM-1) draft genome. Mapped genes were also annotated with Arabidopsis homeolog genes and provide their description. The physical locations including chromosome name of each gene are also provided. First column have G. hirsutum (TM-1) gene name followed by Arabidopsis gene name, TAIR 10 gene description, $G$. hirsutum chromosome number, physical location of the gene and types of strand (+/-). (XLSX $3358 \mathrm{~kb})$

Additional file 2: Differentially expressed genes at 15 DPA between MD52ne and MD90ne. This table contains 4005 allotetraploid cotton genes that differentially significantly express between MD52ne and MD90ne in 15 DPA developing fibers. The threshold absolute value of $\log _{2}$ ratio was $\geq 1$ and $p$ value $\leq 0.05$. Comparison of expression level between MD52ne and MD90ne is also included as fold change. First column have G. hirsutum (TM-1) gene name followed by Arabidopsis gene name, TAIR 10 gene description, $\log _{2}$ ratio of reads per kilo-base per million reads (RPKM) between MD52ne and MD90ne, corresponded $p$ value, adjusted $p$ value and fold change (MD52ne over MD90ne). (XLSX $428 \mathrm{~kb})$

Additional file 3: Differentially expressed genes at 20 DPA between MD52ne and MD90ne. This table contains 1080 allotetraploid cotton genes that differentially significantly express between MD52ne and MD90ne in 20 DPA developing fibers. The threshold absolute value of $\log _{2}$ ratio was $\geq 1$ and $p$ value $\leq 0.05$. Comparison of expression level between MD52ne and MD90ne is also included as fold change. First column have $G$. hirsutum (TM-1) gene name followed by Arabidopsis gene name, TAIR 10 gene description, $\log _{2}$ ratio of reads per kilo-base per million reads (RPKM) between MD52ne and MD90ne, corresponded $p$ value, adjusted $p$ value and fold change (MD52ne over MD90ne). (XLSX 127 kb)
Additional file 4: Differentially expressed up-regulated genes at 15 DPA in MD52ne. Three thousand five hundred sixty five differentially expressed up-regulated genes at 15 DPA in MD52ne are included in this table. First column have G. hirsutum (TM-1) gene name followed by Arabidopsis gene name, TAIR 10 gene description, $\log _{2}$ ratio of reads per kilo-base per million reads (RPKM) between MD52ne and MD90ne, corresponded $p$ value, adjusted $p$ value and fold change (MD52ne over MD90ne). (XLSX $371 \mathrm{~kb}$ )

Additional file 5: Differentially expressed down-regulated genes at 15 DPA in MD52ne. Four hundred forty differentially expressed upregulated genes at 15 DPA in MD90ne are included in this table. First column have $G$. hirsutum (TM-1) gene name followed by Arabidopsis gene name, TAIR 10 gene description, $\log _{2}$ ratio of reads per kilo-base per million reads (RPKM) between MD52ne and MD90ne, corresponded $p$ value, adjusted $p$ value and fold change (MD52ne over MD90ne). (XLSX $63 \mathrm{~kb}$ )

Additional file 6: Differentially expressed up-regulated genes at 20 DPA in MD52ne. Seven hundred seventy four differentially expressed up-regulated genes at 20 DPA in MD52ne are included in this table. First column have G. hirsutum (TM-1) gene name followed by Arabidopsis gene name, TAIR 10 gene description, $\log _{2}$ ratio of reads per kilo-base per million reads (RPKM) between MD52ne and MD90ne, corresponded $p$ value, adjusted $p$ value and fold change (MD52ne over MD90ne). (XLSX $91 \mathrm{~kb}$ )

Additional file 7: Differentially expressed down-regulated genes at 20 DPA in MD52ne. Three hundred six differentially expressed upregulated genes at 15 DPA in MD90ne are included in this table. First column have G. hirsutum (TM-1) gene name followed by Arabidopsis gene name, TAIR 10 gene description, $\log _{2}$ ratio of reads per kilo-base per million reads (RPKM) between MD52ne and MD90ne, corresponded $p$ value, adjusted $p$ value and fold change (MD52ne over MD90ne). (XLSX $42 \mathrm{~kb}$ )

Additional file 8: GO analysis. Singular enrichment analysis was used to identify GO categories that were differentially expressed at 15 (A) and 20 (B) DPA developing fibers from MD52ne. The color and numbers adjacent to the GO identifier represent $p$-values. This file contains the results of $\mathrm{GO}$ enrichment analysis using differentially expressed genes at 15 and 20 DPA separately. (DOCX $198 \mathrm{~kb}$ )

Additional file 9: Differential expressions of receptor like kinases ( $R L K s)$ in developing MD52ne fibers at 20 DPA. Differential expressions of RLKs in different classes were generated by MapMan. Red and purple color represent up and down regulated, respectively. The RLKs contain three domains including extracellular domain, transmembrane (TM), and kinase domain in a cytoplasmic side. C-lectin, RLKs with lectin-like motifs; Crinkly4-like, RLKs with crinkly4-like domains; DUF26, domain of unknown function 26; Extensin, RLK with extensin motif; L-lectin, RLKs with lectin-binding domains; LRK 10-like, RLK gene on Lr10 locus; LRR, leucinerich repeats; LysM, RLKs with lysine motif; PERK-like, proline-rich extensin-like kinase; RKF3-like, receptor-like kinase in flowers 3; S-locus, RLK with Sdomain; Thaumatin, RLK-like thaumatin protein; WAK, wall-associated kinase. This file contains the results of MapMan analysis of different RLKs using differentially expressed genes at 20 DPA. (DOCX $135 \mathrm{~kb}$ )

Additional file 10: RT-qPCR validation of some selected differentially expressed genes from RNA-seq data during fiber development. This file contains the results of qPCR analysis and RNA-seq expression data performed on 32 genes that were used to compare fold expression levels between MD52ne and MD90ne. Four (10, 15, 20 and 24 DPA) and two (15 and 20 DPA) developing fiber samples were used for RT-qPCR and RNA-seq, respectively. RT-qPCR values were corrected to Tubilin and $18 \mathrm{~S}$ gene for each sample. For each treatment group three qPCR measurements were taken for each of three biological replicates and then averaged. (DOCX $644 \mathrm{~kb}$ )

Additional file 11: Names of the important genes related to cotton fiber cell wall development are located near the identified QTL regions. This table contains differentially expressed genes from RNA-seq data that physically located near the two previously reported QTLs associated with BFS and UHML located on chromosomes 03 and 24, respectively by Islam et al. 2014 [49]. (XLSX $12 \mathrm{~kb}$ )

Additional file 12: Comparison of deferentially expressed genes between RNA-seq and microarray of Hinchliffe et al. 2010. These figure compare the number of differential expressed genes between 
RNA-seq (RNA) results of this study and microarray (MA) data reported by Hinchliffe et al. 2010 [17]. RNA-seq and microarray data were generated from 15, 20 and 16, 20 DPA developing fiber samples. A) Total DE genes at both time point; B) DE only expressed in 15 DPA for RNA seq and 16 DPA for microarray data; C) DE only expressed in 20 DPA for both RNA seq and microarray data. (DOCX $320 \mathrm{~kb}$ )

Additional file 13: Cotton MD52ne transcript levels compared with Arabidopsis genes that are co-expressed at secondary wall biogenesis. This table contains 64 secondary cell wall (SCW) biosynthesis related genes retrieved from Arabidopsis genes from PlaNet [67] that are temporally and spatially co-expressed during SCW biosynthesis in Arabidopsis. Those 64 genes were up-regulated in MD52ne. (DOCX $20 \mathrm{~kb}$ )

\section{Additional file 14: Crossing scheme for developing upland cotton} near isgenic lines MD52ne and MD90ne. This figure contains crossing scheme for developing upland cotton near isogenic lines MD52ne and MD90ne has taken from Islam et al. 2014 [50]. In parentheses R is for recurrent and $\mathrm{D}$ is for donor parent for the respective cross. JCPC, DP, MD and FTA are the germplasm name and stand for John Cotton Poly Cross, Deltapine, Mississippi Delta and ARS strain from Pee Dee experiment station, Florescence, SC. (DOCX $131 \mathrm{~kb})$

Additional file 15: Primer name and their sequences used in this study for RT-qPCR validation. This file contains 32 RT-qPCR primer pair sequences that were used to validate RNAseq expression data. Corresponding gene name and descriptions are also included here. (XLSX $13 \mathrm{~kb})$

\section{Abbreviations}

AFIS: Advanced fiber information system; CTL: Chitinase-like;

DEG: Differentially expressed gene; DPA: Days post anthesis; FBS: Fiber bundle strength; FDR: False discovery rate; FE: Fiber elongation; FTIR: Fourier transform infrared spectroscopic; GF: Gravimetric fineness; HVI: Heavy Volume Instrument; GO: Gene ontology; MIC: Micronaire; NIL: Near-isogenic line; PCW: Primary cell wall; RPKM: Reads per kilobase per million reads; RTqPCR: Reverse transcription quantitative polymerase chain reaction; SCW: Secondary cell wall; TF: Transcription factor; UHML: Upper-half mean length.

\section{Competing interests}

The research described in this manuscript was in part funded by a grant from Cotton Incorporated. The funder had no role in study design, data collection and analysis, decision to publish, or preparation of the manuscript. This does not alter the authors' adherence to all polices on sharing data and materials.

\section{Authors' contributions}

MSI collected fiber samples, extracted RNAs, filtered RNA-seq data, performed qPCR validation, analyzed transcriptome data, and wrote the manuscript. DDF conceived the research, designed the experiments, and revised the manuscript. GNT analyzed RNA-seq data and revised the manuscript. CDD was responsible for fiber property measurement. YL measured fiber properties using ATR-FTIR spectroscopy. HJK designed the experiments, coordinated functional and fiber property analyses, analyzed the results, and wrote the manuscript. Al authors read and approved the final manuscript.

\section{Acknowledgements}

This research is dedicated to late USDA-ARS scientist Dr. William R. Meredith Jr. who developed the cotton lines MD90ne and MD52ne at Stoneville, MS, and made available for the present research. Our appreciation goes to Dr. Linghe Zeng of USDA-ARS-SEA for providing AFIS data, Ms. Ping Lee, Tracy Condon and Dr. Xianliang Song for their assistance during fiber sample preparation and Ms. Holly King, Jeannine Moraitis, and Raisa Moiseyev for fiber property analyses. Mention of trade names or commercial products in this article is solely for the purpose of providing specific information and does not imply recommendation or endorsement by the U. S. Department of Agriculture that is an equal opportunity provider and employer.

\section{Funding}

This project was supported by USDA-ARS CRIS project \#6054-21000-017-00D and Cotton Incorporated grant \#12-199.

\section{Author details}

'USDA-ARS, Southern Regional Research Center, Cotton Fiber Bioscience Research Unit, New Orleans, LA 70124, USA. ${ }^{2}$ USDA-ARS, Southern Regional Research Center, Cotton Chemistry and Utilization Research Unit, New Orleans, LA 70124, USA. ${ }^{3}$ USDA-ARS, Southern Regional Research Center, Cotton Structure and Quality Research Unit, New Orleans, LA 70124, USA.

Received: 16 October 2015 Accepted: 28 January 2016

Published online: 01 February 2016

\section{References}

1. Wakelyn PJ, Bertoniere NR, French AD, Thibodeaux DP, Triplett BA, Rousselle M-A, et al. Cotton fiber chemistry and technology, vol. 17. New York, USA: CRC Press; 2010

2. Nichols N, Martin V, Devine J, Li H, Jones D, Hake K. Variety performance: a critical issue for cotton competitiveness. Raliegh, North Carolina: Cotton Incorporated; 2012.

3. Haigler C. Physiological and anatomical factors determining fiber structure and utility. In: Physiology of cotton. New York, USA: Springer; 2010. p. 33-47.

4. Bradow JM, Davidonis GH. Quantitation of fiber quality and the cotton production-processing interface: A physiologist's perspective. J Cotton Sci. 2000:4:34-64.

5. Frydrych I, Thibodeaux DP. Fiber quality evaluation-current and future trends/ intrinsic value of fiber quality in cotton. In: Wakelyn PJ, Chaudhry MR, editors. Cotton: technology for the 21st century. Washington DC: International Cotton Advisory Committee; 2010. p. 251-96.

6. Taylor RA. High speed measurements of strength and elongation. In: World Cotton Research Conference I: 1994; Brisbane, Australia. 1994. p. 268-73.

7. Suh MW, Cui X, Sasser PE. Small bundle tensile properties of cotton related to MANTIS and HVI data - a road to yarn strength prediction. In: Proceeding of Beltwide Cotton Conference: 1996. Nashville, TN: National Cotton Council; 1996. p. 1296-300.

8. Hsieh $Y$-L, Honik E, Hartzell M. A developmental study of single fiber strength: greenhouse grown SJ-2 Acala cotton. Text Res J. 1995;65(2):101-12.

9. Mathangadeera R. Evaluating the impact of fiber processing on cotton fiber tensile properties. Lubbock, TX, USA: Texas Tech University; 2014.

10. Hsieh Y-L. Structural development of cotton fibers and linkages to fiber quality. In: Basra AS, editor. Cotton Fibers Developmental Biology, Quality Improvement, and Textile Processing. New York: Haworth Press, Inc; 1999. p. 137-65.

11. Naylor GR, Delhom CD, Cui X, Gourlot J-P, Rodgers J. Understanding the influence of fiber length on the High Volume Instrument ${ }^{\mathrm{TM}}$ measurement of cotton fiber strength. Text Res J. 2014;84(9):979-88.

12. Munro JM. Cotton. 2nd ed. Harlow, UK: Longman Scientific \& Technical; 1987.

13. Patil NB, Singh M. Development of mediumstaple high-strength cotton suitable for rotor spinning systems. In: World Cotton Conference I: 1995; Bribane, Australia. 1995.

14. Meredith W. Registration of MD 52ne high fiber quality cotton germplasm and recurrent parent MD 90ne. Crop Sci. 2005;45:807-8.

15. Meredith W. Minimum number of genes controlling cotton fiber strength in a backcross population. Crop Sci. 2005;45(3):1114-9.

16. Udall JA, Flagel LE, Cheung F, Woodward AW, Hovav R, Rapp RA, et al. Spotted cotton oligonucleotide microarrays for gene expression analysis. BMC Genomics. 2007:8(1):81.

17. Hinchliffe DJ, Meredith WR, Yeater KM, Kim HJ, Woodward AW, Chen ZJ, et al. Near-isogenic cotton germplasm lines that differ in fiber-bundle strength have temporal differences in fiber gene expression patterns as revealed by comparative high-throughput profiling. Theor Appl Genet. 2010;120(7): 1347-66.

18. Fang L, Tian R, Chen J, Wang S, Li X, Wang P, et al. Transcriptomic analysis of fiber strength in upland cotton chromosome introgression lines carrying different Gossypium barbadense chromosomal segments. PLoS ONE. 2014;9(4):e94642

19. Fang L, Tian R, Li X, Chen J, Wang S, Wang P, et al. Cotton fiber elongation network revealed by expression profiling of longer fiber lines introgressed with different Gossypium barbadense chromosome segments. BMC Genomics. 2014;15(1):838.

20. Kim HJ, Tang Y, Moon HS, Delhom CD, Fang DD. Functional analyses of cotton (Gossypium hirsutum L.) immature fiber (im) mutant infer that fibe cell wall development is associated with stress responses. BMC Genomics. 2013;14(1):889. 
21. Wang C, Lv Y, Xu W, Zhang T, Guo W. Aberrant phenotype and transcriptome expression during fiber cell wall thickening caused by the mutation of the Im gene in immature fiber (im) mutant in Gossypium hirsutum L. BMC Genomics. 2014;15(1):94.

22. Pearson K. Contributions to the Mathematical Theory of Evolution. III. Regression, Heredity, and Panmixia. Proc R Soc Lond. 1895;59(353-358):69-71.

23. Liu Y, Thibodeaux D, Gamble G. Development of Fourier transform infrared spectroscopy in direct, non-destructive, and rapid determination of cotton fiber maturity. Text Res J. 2011;81(15):1559-67.

24. Liu Y, Thibodeaux D, Gamble G, Bauer P, VanDerveer D. Comparative investigation of Fourier transform infrared (FT-IR) spectroscopy and X-ray Diffraction (XRD) in the determination of cotton fiber crystallinity. Appl Spectrosc. 2012;66(8):983-6.

25. Liu Y, Kim HJ. Use of ATR-FTIR spectroscopy in direct, non-destructive, and rapid assessment of developmental cotton fibers grown in planta and in culture. Appl Spectrosc. 2015;69(8):1004-10.

26. Zhang T, Hu Y, Jiang W, Fang L, Guan X, Chen J, et al. Sequencing of allotetraploid cotton (Gossypium hirsutum L. acc. TM-1) provides a resource for fiber improvement. Nat Biotechnol. 2015:33:531-7.

27. Audic S, Claverie J-M. The significance of digital gene expression profiles. Genome Res. 1997;7(10):986-95.

28. Du Z, Zhou X, Ling Y, Zhang Z, Su Z. agriGO: a GO analysis toolkit for the agricultural community. Nucleic Acids Res. 2010;38(Web Server):W64-70.

29. Shi Y-H, Zhu S-W, Mao X-Z, Feng J-X, Qin Y-M, Zhang L, et al. Transcriptome profiling, molecular biological, and physiological studies reveal a major role for ethylene in cotton fiber cell elongation. Plant Cell. 2006;18(3):651-64.

30. Li G, Meng X, Wang R, Mao G, Han L, Liu Y, et al. Dual-level regulation of ACC synthase activity by MPK3/MPK6 cascade and its downstream WRKY transcription factor during ethylene induction in Arabidopsis. PLoS Genet. 2012;8(6):e1002767.

31. Binder BM, Walker JM, Gagne JM, Emborg TJ, Hemmann G, Bleecker AB, et al. The Arabidopsis EIN3 binding F-Box proteins EBF1 and EBF2 have distinct but overlapping roles in ethylene signaling. Plant Cell. 2007;19(2):509-23.

32. Rubinovich $\mathrm{L}$, Weiss $\mathrm{D}$. The Arabidopsis cysteine-rich protein GASA4 promotes GA responses and exhibits redox activity in bacteria and in planta. Plant J. 2010;64(6):1018-27.

33. Hamann $\mathrm{T}$. The plant cell wall integrity maintenance mechanism-A case study of a cell wall plasma membrane signaling network. Phytochemistry. 2015;112:100-9.

34. Xu S-L, Rahman A, Baskin TI, Kieber JJ. Two leucine-rich repeat receptor kinases mediate signaling, linking cell wall biosynthesis and ACC synthase in Arabidopsis. Plant Cell. 2008;20(11):3065-79.

35. Harpaz-Saad S, McFarlane HE, Xu S, Divi UK, Forward B, Western TL, et al. Cellulose synthesis via the FEI2 RLK/SOS5 pathway and cellulose synthase 5 is required for the structure of seed coat mucilage in Arabidopsis. Plant $\mathrm{J}$. 2011;68(6):941-53.

36. An C, Saha S, Jenkins JN, Scheffler BE, Wilkins TA, Stelly DM. Transcriptome profiling, sequence characterization, and SNP-based chromosomal assignment of the EXPANSIN genes in cotton. Mol Gen Genomics. 2007;278(5):539-53.

37. Ruan Y-L, Llewellyn DJ, Furbank RT. The control of single-celled cotton fiber elongation by developmentally reversible gating of plasmodesmata and coordinated expression of sucrose and $\mathrm{K}+$ transporters and expansin. Plant Cell Online. 2001;13(1):47-60.

38. Liu Q, Talbot M, Llewellyn DJ. Pectin methylesterase and pectin remodelling differ in the fibre walls of two gossypium species with very different fibre properties. PLOS ONE. 2013;8(6):e65131.

39. Lee J, Burns TH, Light G, Sun Y, Fokar M, Kasukabe Y, et al. Xyloglucan endotransglycosylase/hydrolase genes in cotton and their role in fiber elongation. Planta. 2010;232(5):1191-205.

40. Jiang Y, Guo W, Zhu H, Ruan Y-L, Zhang T. Overexpression of GhSusA1 increases plant biomass and improves cotton fiber yield and quality. Plant Biotechnol J. 2012;10(3):301-12

41. Kim HJ, Hinchliffe DJ, Triplett BA, Chen ZJ, Stelly DM, Yeater KM, et al. Phytohormonal networks promote differentiation of fiber initials on pre-anthesis cotton ovules grown in vitro and in planta. PLoS One. 2015;10(4):e0125046.

42. Liu L, Shang-Guan K, Zhang B, Liu X, Yan M, Zhang L, et al. Brittle Culm1, a COBRA-like protein, functions in cellulose assembly through binding cellulose microfibrils. PLoS Genet. 2013;9(8):15.

43. Sánchez-Rodríguez $C$, Bauer $S$, Hématy $K$, Saxe F, Ibáñez AB, Vodermaier $V$, et al. Chitinase-like1/pom-pom1 and its homolog CTL2 are glucan- interacting proteins important for cellulose biosynthesis in Arabidopsis. Plant Cell. 2012;24(2):589-607.

44. Zhong R, Ye ZH. MYB46 and MYB83 bind to the SMRE sites and directly activate a suite of transcription factors and secondary wall biosynthetic genes. Plant Cell Physiol. 2012;53(2):368-80.

45. Yang C, Xu Z, Song J, Conner K, Barrena GV, Wilson ZA. Arabidopsis MYB26/ MALE STERILE35 regulates secondary thickening in the endothecium and is essential for anther dehiscence. Plant Cell. 2007;19(2):534-48.

46. Ko J-H, Jeon H-W, Kim W-C, Kim J-Y, Han K-H. The MYB46/MYB83-mediated transcriptional regulatory programme is a gatekeeper of secondary wall biosynthesis. Ann Bot. 2014;114(6):1099-107. mcu126.

47. Kim HJ, Murai N, Fang DD, Triplett BA. Functional analysis of Gossypium hirsutum cellulose synthase catalytic subunit 4 promoter in transgenic Arabidopsis and cotton tissues. Plant Sci. 2011;180(2):323-32.

48. Mokshina N, Gorshkova T, Deyholos MK. Chitinase-like (CTL) and cellulose synthase (CESA) gene expression in gelatinous-type cellulosic walls of flax (Linum usitatissimum L.) bast fibers. PLoS ONE. 2014:9(6):e97949.

49. Islam MS, Zeng L, Delhom CD, Song X, Kim HJ, Li P, et al. Identification of cotton fiber quality quantitative trait loci using intraspecific crosses derived from two near-isogenic lines differing in fiber bundle strength. Molecular Breeding 2014:1-12.

50. Li F, Fan G, Lu C, Xiao G, Zou C, Kohel RJ, et al. Genome sequence of cultivated Upland cotton (Gossypium hirsutum TM-1) provides insights into genome evolution. Nat Biotechnol. 2015;33(5):524-30.

51. Seagull RW. Cytoskeletal involvement in cotton fiber growth and development. Micron. 1993;24(6):643-60.

52. Haigler $\mathrm{CH}$, Betancur L, Stiff MR, Tuttle JR. Cotton fiber: a powerful singlecell model for cell wall and cellulose research. Frontiers Plant Sci. 2012;3:104.

53. Benedict CR, Kohel JR, Lewis HL. Cotton fiber quality. In: Smith CW, Cothren JT, editors. Cotton origin, histrory, technology, and production. New York: John Wiley \& sons, Inc; 1999. p. 269-88.

54. Kim HJ. Fiber biology. In: Fang DD, Percy RG, Madison WI, editors. American Society of Agronomy, Crop Science Society of America, and Soil Science Society of America. 2nd ed. 2015. p. 97-127.

55. Beasley C, Ting IP. Effects of plant growth substances on in vitro fiber development from unfertilized cotton ovules. Am J Bot. 1974;61:188-94.

56. Singh B, Avci U, Inwood SEE, Grimson MJ, Landgraf J, Mohnen D, et al. A specialized outer layer of the primary cell wall joins elongating cotton fibers into tissue-like bundles. Plant Physiol. 2009;150(2):684-99.

57. De Smet I, Voß U, Jürgens G, Beeckman T. Receptor-like kinases shape the plant. Nat Cell Biol. 2009;11(10):1166-73.

58. Yoon GM, Kieber JJ. 1-Aminocyclopropane-1-carboxylic acid as a signalling molecule in plants. AoB Plants. 2013;5:plt017.

59. Wang J, Kucukoglu M, Zhang L, Chen P, Decker D, Nilsson O, et al. The Arabidopsis LRR-RLK, PXC1, is a regulator of secondary wall formation correlated with the TDIF-PXY/TDR-WOX4 signaling pathway. BMC Plant Biol. 2013;13(1):94

60. Song D, Xi W, Shen J, Bi T, Li L. Characterization of the plasma membrane proteins and receptor-like kinases associated with secondary vascular differentiation in poplar. Plant Mol Biol. 2011;76(1-2):97-115.

61. Li Y-L, Sun J, Xia G-X. Cloning and characterization of a gene for an LRR receptor-like protein kinase associated with cotton fiber development. Mol Gen Genomics. 2005;273(3):217-24.

62. Ben-Tov D, Abraham Y, Stav S, Thompson K, Loraine A, Elbaum R, et al. COBRA-LIKE 2, a member of the GPI-anchored COBRA-LIKE family, plays a role in cellulose deposition in Arabidopsis seed coat mucilage secretory cells. Plant Physiol. 2015;167(3):711-24.

63. Li Y, Qian Q, Zhou Y, Yan M, Sun L, Zhang M, et al. BRITTLE CULM1, which encodes a COBRA-like protein, affects the mechanical properties of rice plants. Plant Cell. 2003;15(9):2020-31.

64. Ching A, Dhugga KS, Appenzeller L, Meeley R, Bourett TM, Howard RJ, et al. Brittle stalk 2 encodes a putative glycosylphosphatidylinositolanchored protein that affects mechanical strength of maize tissues by altering the composition and structure of secondary cell walls. Planta. 2006;224(5):1174-84.

65. Bravo JM, Campo S, Murillo I, Coca M, San Segundo B. Fungus-and woundinduced accumulation of mRNA containing a class II chitinase of the pathogenesis-related protein 4 (PR-4) family of maize. Plant Mol Biol. 2003;52(4):745-59.

66. Nakazaki T, Tsukiyama T, Okumoto Y, Kageyama D, Naito K, Inouye K, et al. Distribution, structure, organ-specific expression, and phylogenic analysis of 
the pathogenesis-related protein-3 chitinase gene family in rice (Oryza sativa L.). Genome. 2006;49(6):619-30.

67. Mutwil M, Klie S, Tohge T, Giorgi FM, Wilkins O, Campbell MM, et al. PlaNet: combined sequence and expression comparisons across plant networks derived from seven species. Plant Cell. 2011;23(3):895-910.

68. Delhom CD, Cui X, Thibodeaux D. Single fiber testing via Favimat. Proceedings of Beltwide Cotton Conference 2010:1405-1410.

69. American Society for Testing and Materials. Standard test method for linear density of textile fibers. Option A, Fiber bundle weighing. In.: ASTM Standard D1577-07. Annu. Book of ASTM Standards. Philadelphia, PA: ASTM; 2012.

70. Boylston EK, Thibodeaux DP, Evans JP. Applying Microscopy to the Development of a Reference Method for Cotton Fiber Maturity. Textile Res J. 1993;63(2):80-7.

71. Xu B, Huang Y. Image Analysis for Cotton Fibers Part II: Cross-Sectional Measurements. Text Res J. 2004;74(5):409-16.

72. Thibodeaux DP, Evans JP. Cotton fiber maturity by image analysis. Text Res J. 1986;56(2):130-9.

73. Thibodeaux DP, Rajasekaran K. Development of new reference standards for cotton fiber maturity. J Cotton Sci. 1999;3:188-93.

74. Naoumkina M, Thyssen G, Fang DD, Hinchliffe DJ, Florane C, Yeater KM, et al. The Li2 mutation results in reduced subgenome expression bias in elongating fibers of allotetraploid cotton (Gossypium hirsutum L.) PLoS One. 2014:9(3):e90830.

75. Wu TD, Nacu S. Fast and SNP-tolerant detection of complex variants and splicing in short reads. Bioinformatics. 2010;26(7):873-81.

76. Quinlan AR, Hall IM. BEDTools: a flexible suite of utilities for comparing genomic features. Bioinformatics. 2010;26(6):841-2.

77. Robinson MD, McCarthy DJ, Smyth GK. edgeR: a Bioconductor package for differential expression analysis of digital gene expression data. Bioinformatics. 2010;26(1):139-40.

78. Hulsen T, de Vlieg J, Alkema W. BioVenn-a web application for the comparison and visualization of biological lists using area-proportional Venn diagrams. BMC Genomics. 2008;9(1):488

79. Thimm O, Bläsing O, Gibon Y, Nagel A, Meyer S, Krüger P, et al. mapman: a user-driven tool to display genomics data sets onto diagrams of metabolic pathways and other biological processes. Plant J. 2004;37(6):914-39.

\section{Submit your next manuscript to BioMed Central and we will help you at every step:}

- We accept pre-submission inquiries

- Our selector tool helps you to find the most relevant journal

- We provide round the clock customer support

- Convenient online submission

- Thorough peer review

- Inclusion in PubMed and all major indexing services

- Maximum visibility for your research

Submit your manuscript at www.biomedcentral.com/submit

) Biomed Central 